\title{
EQUIVALENT CROSSED PRODUCTS OF MONOIDAL HOM-HOPF ALGEBRAS
}

\author{
Zhongwei Wang, Liangyun Zhang and Huinui Zheng \\ Jinling Institute of Technology, China and Nanjing Agricultural University, \\ China
}

\begin{abstract}
In this paper, we give a Maschke-type theorem for a Hom-crossed product on a finite dimensional monoidal Hom-Hopf algebra, and investigate a sufficient and necessary condition for two Hom-crossed products to be equivalent. Furthermore, we construct an equivalent Homcrossed system based on a same Hom-crossed product by using lazy Hom2-cocyle.
\end{abstract}

\section{INTRODUCTION}

Hom-type algebras appeared first in physical contexts, in connection with twisted, discretized or deformed derivatives and corresponding generalizations, discretizations and deformations of vector fields and differential calculus. They were introduced in the form of Hom-Lie algebras in [10], where the Jacobi identity was twisted along a linear endomorphism, namely

$$
[\alpha(x),[y, z]]+[\alpha(y),[z, x]]+[\alpha(z),[x, y]]=0,
$$

where $\alpha$ is an endomorphism of the Hom-Lie algebra (see [7, 11, 18]). Meanwhile, Hom-associative algebras have been suggested in [13]. Consequently, other Hom-type structures such as Hom-coalgebras, Hom-bialgebras, HomHopf algebras have been introduced and investigated in $[9,14,19]$. The socalled twisting principle that was introduced in [17] to provide Hom-type generalization of algebras has been used to obtain many more properties of Hom-bialgebras and Hom-Hopf algebras.

The authors of [2] investigated the counterparts of Hom-bialgebras and Hom-Hopf algebras in the context of tensor categories, and termed them

2010 Mathematics Subject Classification. 16T05, 18D10, 16T25.

Key words and phrases. Monoidal Hom-Hopf algebra, Hom-crossed product, Hom-cleft extension, Maschke-type theorem, Hom-crossed system, lazy Hom-2-cocyle. 
monoidal Hom-bialgebras and monoidal Hom-Hopf algebras with slight variations in their definitions. Consequently, the antipodes, integrals and Drinfel'd doubles of Hom-Hopf algebras were considered in $[6,16]$. Further, some modules and comodules on these Hom-algebras structures such as Hom-module algebras, Hom-comodule algebras and Hom-Hopf modules were considered in $[5,4,3,19]$.

It is known that the theory of Hopf Galois extensions which roots from the Galois theory for groups acting on commutative rings, plays an important role in the theory of Hopf algebras. Therefore, their Hom-cases were introduced in $[5,12]$. As a special case of the Hopf Galois extension, a cleft extension can be characterized as a crossed product with a convolution cocycle by a Hopf algebra, and the Hom-case parallelled holds as well (see [12]). In [8], Doi introduced an equivalence of crossed systems and considered the problem of determining when two cleft extensions are isomorphic. Hence, it is natural to investigate the equivalence of Hom-crossed products. This is our main motivation.

This paper is organized as follows. In section 2 , we recall some definitions and results on monoidal Hom-Hopf algebras. In section 3, we give differentiated conditions for a Hom-crossed product to be a Hom-cleft extension. As an application, we give a Maschke-type theorem for the Hom-crossed product on a finite dimensional monoidal Hom-Hopf algebra which equips a right integral. In section 4, we introduce the conception of equivalent Hom-crossed systems, and investigate a sufficient and necessary condition for two Hom-crossed products to be equivalent. Meanwhile, we construct an equivalent Hom-crossed system based on a same Hom-crossed product, by using lazy Hom-2-cocyles.

Throughout this article, all the vector spaces, tensor products and homomorphisms are over a fixed field $k$ unless otherwise stated. We use the Sweedler's notations for the terminologies on coalgebras and comodules. For a coalgebra $C$, we write Hom-comultiplication $\Delta(c)=c_{1} \otimes c_{2}$ for any $c \in C$, and for a right $C$-comodule $M$, we denote its coaction by $\rho(m)=m_{(0)} \otimes m_{(1)}$ for any $m \in M$.

\section{Preliminaries}

In this section we recall from [2] some informations about so-called monoidal Hom-structures. Briefly speaking, these structures are objects in the monoidal category $\widetilde{\mathcal{H}}\left(\mathcal{M}_{k}\right)$ (called the Hom-category associated to $\mathcal{M}_{k}$ in $[2])$, where $\mathcal{M}_{k}=\left(\mathcal{M}_{k}, \otimes, k, a, l, r\right)$ is the category of $k$-modules. And the

category $\widetilde{\mathcal{H}}\left(\mathcal{M}_{k}\right)$ is defined as follows: the objects are couples $(M, \mu)$, with $M \in \mathcal{M}_{k}$ and $\mu \in \operatorname{Aut}_{k}(M)$, the morphism $f:(M, \mu) \rightarrow(N, \nu)$ is a morphism $f: M \rightarrow N$ in $\mathcal{M}_{k}$ such that $\nu \circ f=f \circ \mu$, and the monoidal product of $(M, \mu)$ and $(N, \nu)$ is given in [7] by

$$
(M, \mu) \otimes(N, \nu)=(M \otimes N, \mu \otimes \nu), \text { and }(k, i d),
$$


EQUIVALENT CROSSED PRODUCTS OF MONOIDAL HOM-HOPF ALGEBRAS 347

with the associator

$$
\widetilde{a}_{M, N, L}=a_{M, N, L} \circ\left((\mu \otimes i d) \otimes \varsigma^{-1}\right)=\left(\mu \otimes\left(i d \otimes \varsigma^{-1}\right)\right) \circ a_{M, N, L},
$$

and the unitors

$$
\widetilde{l}_{M}=\mu \circ l_{M}=l_{M} \circ(i d \otimes \mu), \quad \widetilde{r}_{M}=\mu \circ r_{M}=r_{M} \circ(\mu \otimes i d),
$$

for any $(M, \mu),(N, \nu),(L, \varsigma) \in \widetilde{\mathcal{H}}\left(\mathcal{M}_{k}\right)$.

A $k$-submodule $N \subseteq M$ is called a subobject of $(M, \mu)$ if $\mu$ restricts to $N$ being an automorphism of $N$, that is, $\left(N,\left.\mu\right|_{N}\right) \in \widetilde{\mathcal{H}}\left(\mathcal{M}_{k}\right)$. $\widetilde{\mathcal{H}}\left(\mathcal{M}_{k}\right)$.

In the following, our considered objects are in the monoidal category

Definition 2.1 ([2]). (1) A monoidal Hom-algebra is an object $(A, \alpha)$ $\in \widetilde{\mathcal{H}}\left(\mathcal{M}_{k}\right)$ together with an element $1_{A} \in A$ (called unit) and linear maps $m: A \otimes A \rightarrow A ; a \otimes b \mapsto a b$ and $\alpha \in A_{u} t_{k}(A)$ such that

$$
\begin{gathered}
\alpha(a)(b c)=(a b) \alpha(c), \quad \alpha(a b)=\alpha(a) \alpha(b), \\
a 1_{A}=1_{A} a=\alpha(a), \quad \alpha\left(1_{A}\right)=1_{A},
\end{gathered}
$$

for all $a, b, c \in A$.

(2) A monoidal Hom-coalgebra is an object $(C, \gamma) \in \widetilde{\mathcal{H}}\left(\mathcal{M}_{k}\right)$ together with linear maps $\Delta: C \rightarrow C \otimes C, \Delta(c)=c_{1} \otimes c_{2}$ and $\varepsilon: C \rightarrow k$ such that

$$
\begin{gathered}
\gamma^{-1}\left(c_{1}\right) \otimes c_{21} \otimes c_{22}=c_{11} \otimes c_{12} \otimes \gamma^{-1}\left(c_{2}\right), \quad \Delta(\gamma(c))=\gamma\left(c_{1}\right) \otimes \gamma\left(c_{2}\right), \\
c_{1} \varepsilon\left(c_{2}\right)=\gamma^{-1}(c)=\varepsilon\left(c_{1}\right) c_{2}, \quad \varepsilon(\gamma(c))=\varepsilon(c),
\end{gathered}
$$

for all $c \in C$.

If $\left(A, m_{A}, \eta_{A}, \alpha\right)$ is a monoidal Hom-algebra, and $\left(C, \Delta_{C}, \varepsilon_{C}, \gamma\right)$ is a monoidal Hom-coalgebra, then it was shown in [2, Proposition 2.9] that $\operatorname{Hom}(C, A)$ has a monoidal Hom-algebra structure under convolution product "*". For any $\phi, \varphi \in \operatorname{Hom}(C, A)$, the convolution product is defined by

$$
\phi * \varphi=m_{A} \circ(\phi \otimes \varphi) \circ \Delta_{C} .
$$

The unit of $\operatorname{Hom}(C, A)$ is $\eta_{A} \circ \varepsilon_{C}$, and the twisting automorphism $\lambda$ is $\lambda(\phi)=$ $\alpha \circ \phi \circ \gamma^{-1}$. For $\phi \in \operatorname{Hom}(C, A)$, if there exists $\phi^{\prime} \in \operatorname{Hom}(C, A)$ such that $\phi * \phi^{\prime}=\phi^{\prime} * \phi=\eta_{A} \circ \varepsilon_{C}$, then we say that $\phi$ is convolution invertible.

Definition 2.2 ([2]). A monoidal Hom-bialgebra $H=(H, \alpha, m, \eta, \Delta, \varepsilon)$ is a bialgebra in the monoidal category $\widetilde{\mathcal{H}}\left(\mathcal{M}_{k}\right)$. This means that $(H, \alpha, m, \eta)$ is a monoidal Hom-algebra and $(H, \alpha, \Delta, \varepsilon)$ is a monoidal Hom-coalgebra such that $\Delta$ and $\varepsilon$ are morphisms of Hom-algebras, that is, for any $h, g \in H$,

$$
\begin{gathered}
\Delta(h g)=\Delta(h) \Delta(g), \Delta\left(1_{H}\right)=1_{H} \otimes 1_{H}, \\
\varepsilon(h g)=\varepsilon(h) \varepsilon(g), \varepsilon\left(1_{H}\right)=1_{k} .
\end{gathered}
$$


A monoidal Hom-bialgebra $(H, \alpha)$ is called monoidal Hom-Hopf algebra if there exists a morphism (called antipode) $S: H \rightarrow H$ in $\widetilde{\mathcal{H}}\left(\mathcal{M}_{k}\right)$ (i.e. $S \circ \alpha=\alpha \circ S)$, such that for all $h \in H$,

$$
S\left(h_{1}\right) h_{2}=\varepsilon(h) 1_{H}=h_{1} S\left(h_{2}\right) .
$$

Note that a monoidal Hom-Hopf algebra is by definition a Hopf algebra in $\widetilde{\mathcal{H}}\left(\mathcal{M}_{k}\right)$. Further, the antipode of a monoidal Hom-Hopf algebra is a monoidal Hom-anti-(co)algebra homomorphism, that is, for any $h, g \in H$,

$$
\begin{gathered}
S(h g)=S(g) S(h), S\left(1_{H}\right)=1_{H}, \\
\Delta(S(h))=S\left(h_{2}\right) \otimes S\left(h_{1}\right), \varepsilon \circ S=\varepsilon .
\end{gathered}
$$

Let $(H, \alpha)$ be a finite dimensional monoidal Hom-Hopf algebra. A right integral in $(H, \alpha)$ (ref. [6]) is an $\alpha$-invariant element $t \in H$ (i.e., $\alpha(t)=t$ ) such that $t h=\varepsilon(h) t$ for all $h \in H$. If $\varepsilon(t)=1$ for a right integral $t$, then $t$ is said to be normalized.

In the following, we recall the actions on monoidal Hom-algebras and coactions on monoidal Hom-coalgebras.

Definition $2.3([2])$. (1) Let $(A, \alpha)$ be a monoidal Hom-algebra. A left $(A, \alpha)$-Hom-module consists of $(M, \mu)$ in $\widetilde{\mathcal{H}}\left(\mathcal{M}_{k}\right)$ together with a morphism $\psi: A \otimes M \rightarrow M, \psi(a \otimes m)=a \cdot m$ such that

$\alpha(a) \cdot(b \cdot m)=(a b) \cdot \mu(m), \quad \mu(a \cdot m)=\alpha(a) \cdot \mu(m), \quad 1_{A} \cdot m=\mu(m)$,

for all $a, b \in A$ and $m \in M$.

For left $(A, \alpha)$-Hom-modules $(M, \mu)$ and $(N, \nu)$, a morphism $f$ : $M \rightarrow N$ in $\widetilde{\mathcal{H}}\left(\mathcal{M}_{k}\right)$ is called left $(A, \alpha)$-linear if $f(a \cdot m)=a \cdot f(m)$ for all $a \in A, m \in M$. In a similar way, we can define a right $(A, \alpha)$ Hom-module and a right $(A, \alpha)$-linear morphism.

(2) Let $(C, \gamma)$ be a monoidal Hom-coalgebra. A right $(C, \gamma)$-Hom-comodule is an object $(M, \mu)$ in $\widetilde{\mathcal{H}}\left(\mathcal{M}_{k}\right)$ together with a $k$-linear map $\rho_{M}: M \rightarrow$ $M \otimes C, \rho_{M}(m)=m_{(0)} \otimes m_{(1)}$ such that

$$
\begin{gathered}
\mu^{-1}\left(m_{(0)}\right) \otimes\left(m_{(1) 1} \otimes m_{(1) 2}\right)=m_{(0)(0)} \otimes\left(m_{(0)(1)} \otimes \gamma^{-1}\left(m_{(1)}\right)\right), \\
\rho_{M}(\mu(m))=\mu\left(m_{(0)}\right) \otimes \gamma\left(m_{(1)}\right), \\
m_{(0)} \varepsilon\left(m_{(1)}\right)=\mu^{-1}(m),
\end{gathered}
$$

for all $m \in M$.

For right $(C, \gamma)$-Hom-comodules $(M, \mu)$ and $(N, \nu)$, a morphism $f: M \rightarrow N$ in $\widetilde{\mathcal{H}}\left(\mathcal{M}_{k}\right)$ is called right $(C, \gamma)$-colinear if $\rho(f(m))=$ $f\left(m_{(0)}\right) \otimes m_{(1)}$ for all $m \in M$.

Let $(H, \alpha)$ be a monoidal Hom-bialgebra, and $(M, \mu)$ is a right $(H, \alpha)$ Hom-comodule with the comodule structure $\rho$. The coinvariant of $(H, \alpha)$ on 
EQUIVALENT CROSSED PRODUCTS OF MONOIDAL HOM-HOPF ALGEBRAS 349

$(M, \mu)$ is the set

$$
M^{c o H}=\left\{m \in M \mid \rho(m)=\nu^{-1}(m) \otimes 1_{H}\right\} .
$$

Definition 2.4. Let $(H, \alpha)$ be a monoidal Hom-bialgebra. A monoidal Hom-algebra $(A, \beta)$ is called an $(H, \alpha)$-Hom-comodule algebra if there is a right $(H, \alpha)$-Hom-comodule structure map $\rho_{A}: A \rightarrow A \otimes H$ on $(A, \beta)$ such that

$$
\begin{gathered}
\rho_{A}\left(1_{A}\right)=1_{A} \otimes 1_{H}, \\
\rho_{A}(a b)=\rho_{A}(a) \rho_{A}(b),
\end{gathered}
$$

for all $a, b \in A$.

Definition 2.5. Let $(H, \alpha)$ be a monoidal Hom-Hopf algebra, and $\left(B,\left.\beta\right|_{B}\right)$ a subobject of an object $(A, \beta) \in \widetilde{\mathcal{H}}\left(\mathcal{M}_{k}\right)$.

(1) $\left(B,\left.\beta\right|_{B}\right) \subseteq(A, \beta)$ is a (right) $(H, \alpha)$-extension if $(A, \beta)$ is a right $(H, \alpha)$-Hom-comodule algebra with $A^{\text {coH }}=B$.

(2) The $(H, \alpha)$-extension $\left(B,\left.\beta\right|_{B}\right) \subseteq(A, \beta)$ is called Hom-cleft if there exists a right $(H, \alpha)$-Hom-comodule map $\lambda:(H, \alpha) \rightarrow(A, \beta)$ which is convolution invertible.

\section{Characterization of Hom-Crossed products and application}

In this section, we characterize a Hom-cleft extension as a Hom-crossed product. As an application, we give a Maschke-type theorem for the Homcrossed product on a finite dimensional monoidal Hom-Hopf algebra which equips a right integral.

First, we recall the following definition.

Definition $3.1([12,15])$. Let $(H, \alpha)$ be a monoidal Hom-bialgebra, and $(A, \beta)$ a monoidal Hom-algebra. We say that $H$ weakly acts on $A$ if there is a $k$-linear map $H \otimes A \rightarrow A, h \otimes a \mapsto h \cdot a$ such that

$$
\begin{gathered}
\beta(h \cdot a)=\alpha(h) \cdot \beta(a), \\
h \cdot 1_{A}=\varepsilon(h) 1_{A}, \\
h \cdot(a b)=\left(h_{1} \cdot a\right)\left(h_{2} \cdot b\right),
\end{gathered}
$$

for all $a, b \in A, h \in H$.

The weak action is said to be inner if there exists a convolution invertible morphism $\lambda:(H, \alpha) \rightarrow(A, \beta)$ in $\widetilde{\mathcal{H}}\left(\mathcal{M}_{k}\right)$ such that

$$
h \cdot a=\left(\lambda\left(h_{1}\right) \beta^{-1}(a)\right) \lambda^{-1}\left(\alpha\left(h_{2}\right)\right),
$$

for all $h \in H, a \in A$.

Let $(H, \alpha)$ be a monoidal Hom-bialgebra, and $(A, \beta)$ a monoidal Homalgebra. Assume that $(H, \alpha)$ weakly acts on $(A, \beta)$ and $\sigma: H \otimes H \rightarrow A$ is a convolution invertible map in $\widetilde{\mathcal{H}}\left(\mathcal{M}_{k}\right)$. The Hom-crossed product $\left(A \#_{\sigma} H, \beta \#_{\sigma} \alpha\right)$ 
is defined as follows: $A \#_{\sigma} H=A \otimes H$ as $k$-spaces, and the Hom-multiplication is given by

$$
\left(a \#_{\sigma} h\right)\left(b \#_{\sigma} g\right)=a\left(\left(\alpha^{-1}\left(h_{1}\right) \cdot \beta^{-2}(b)\right) \sigma\left(h_{21}, \alpha^{-1}\left(g_{1}\right)\right)\right) \#_{\sigma} \alpha^{2}\left(h_{22}\right) \alpha\left(g_{2}\right),
$$

for all $a, b \in A, h, g \in H$.

It is known from [12, Proposition 3.3] that the Hom-crossed product $\left(A \#{ }_{\sigma} H, \beta \#_{\sigma} \alpha\right)$ is a monoidal Hom-algebra with unit $1_{A} \#_{\sigma} 1_{H}$ if and only if the following conditions hold.

(1) $A$ is a twisted $H$-module, that is, $1_{H} \cdot a=\beta(a)$ and

$$
\left(\alpha\left(x_{1}\right) \cdot\left(y_{1} \cdot \beta^{-1}(a)\right)\right) \sigma\left(\alpha\left(x_{2}\right), \alpha\left(y_{2}\right)\right)=\sigma\left(\alpha\left(x_{1}\right), \alpha\left(y_{1}\right)\right)\left(\left(x_{2} y_{2}\right) \cdot a\right),
$$

for all $x, y \in H, a \in A$.

(2) $\sigma$ is a normal Hom-cocycle, that is, $\sigma\left(1_{H}, x\right)=\sigma\left(x, 1_{H}\right)=\varepsilon(x) 1_{A}$ and

$$
\left(\alpha\left(x_{1}\right) \cdot \sigma\left(y_{1}, z_{1}\right)\right) \sigma\left(\alpha\left(x_{2}\right), y_{2} z_{2}\right)=\sigma\left(\alpha\left(x_{1}\right), \alpha\left(y_{1}\right)\right) \sigma\left(x_{2} y_{2}, z\right),
$$

for all $x, y, z \in H$.

In what follows, such pair $(\cdot, \sigma)$ in a Hom-crossed product $\left(A \#_{\sigma} H, \beta \#_{\sigma} \alpha\right)$ is said to be a Hom-crossed system for $(H, \alpha)$ over $(A, \beta)$.

ExAmPle 3.2. (1) Let $\left(A \#_{\sigma} H, \beta \#_{\sigma} \alpha\right)$ be a Hom-crossed product. Consider the case when $\sigma$ is trivial, that is, $\sigma(h, g)=\varepsilon(h) \varepsilon(g) 1_{A}$ for all $h, g \in H$. Then $\left(A \#_{\sigma} H, \beta \#_{\sigma} \alpha\right)$ reduces to a Hom-smash product (see [6]).

(2) If the weak action is trivial, i.e., $h \cdot a=\varepsilon(h) \beta(a)$ for all $h \in H, a \in A$, then we write $A \#_{\sigma} H=A_{\sigma}[H]$, and call the Hom-crossed product a Homtwisted product. The Hom-multiplication of $A_{\sigma}[H]$ is induced by as follows:

$$
\left(a \otimes_{\sigma} h\right)\left(b \otimes_{\sigma} g\right)=\beta^{-1}(a b) \sigma\left(h_{1}, g_{1}\right) \otimes_{\sigma} \alpha\left(h_{2} g_{2}\right) .
$$

for all $a, b \in A, h, g \in H$.

It is clear that the Hom-crossed product $\left(A \#_{\sigma} H, \beta \#_{\sigma} \alpha\right)$ is both a left $(A, \beta)$-module via the following action induced by the Hom-multiplication

$$
a \otimes\left(b \#_{\sigma} h\right) \mapsto a b \#_{\sigma} \alpha(h),
$$

and a right $(H, \alpha)$-comodule via the coaction

$$
\rho_{A \#_{\sigma} H}: a \#_{\sigma} h \mapsto\left(\beta^{-1}(a) \#_{\sigma} h_{1}\right) \otimes \alpha\left(h_{2}\right),
$$

where $a, b \in A, h \in H$.

Lemma 3.3 ([12]). The right $(H, \alpha)$-comodule structure map $\rho_{A \#_{\sigma} H}$ turns $\left(A \#_{\sigma} H, \beta \#_{\sigma} \alpha\right)$ into an $(H, \alpha)$-Hom-comodule algebra. Moreover, the map $\lambda:(H, \alpha) \rightarrow\left(A \#{ }_{\sigma} H, \beta \#_{\sigma} \alpha\right)$ defined by $\lambda(h)=1_{A} \#_{\sigma} \alpha^{-1}(h)$ is a convolution invertible $(H, \alpha)$-Hom-comodule map.

As in Hopf algebra case, the Hom-cleft extensions can be characterized as crossed products with convolution invertible cocycles by Hom-Hopf algebras. 
EQUIVALENT CROSSED PRODUCTS OF MONOIDAL HOM-HOPF ALGEBRAS 351

Theorem 3.4. [12, Thereom 4.5] Let $(H, \alpha)$ be a monoidal Hom-Hopf algebra and $\left(B,\left.\beta\right|_{B}\right) \subseteq(A, \beta)$ an $(H, \alpha)$-extension. Then the following statements are equivalent.

(1) The extension $\left(B,\left.\beta\right|_{B}\right) \subseteq(A, \beta)$ is $(H, \alpha)$-cleft;

(2) There exist a convolution invertible cocycle $\sigma$ and a weak action of $(H, \alpha)$ on $\left(B,\left.\beta\right|_{B}\right)$ such that $(A, \beta)$ is isomorphic to the Homcrossed product $\left(B \#_{\sigma} H,\left.\beta\right|_{B} \#_{\sigma} \alpha\right)$ as left $\left(B,\left.\beta\right|_{B}\right)$-Hom-modules and right $(H, \alpha)$-Hom-comodule algebras.

Proof. Sketch. Suppose that $\left(B,\left.\beta\right|_{B}\right) \subseteq(A, \beta)$ is $(H, \alpha)$-cleft via $\lambda$ : $(H, \alpha) \rightarrow(A, \beta)$. Then the weak action of $(H, \alpha)$ on $\left(B,\left.\beta\right|_{B}\right)$ is given by

$$
h \cdot b=\left(\lambda\left(h_{1}\right) \beta^{-1}(b)\right) \lambda^{-1}\left(\alpha\left(h_{2}\right)\right),
$$

and the Hom-cocycle is defined by

$$
\sigma:(H \otimes H, \alpha \otimes \alpha) \rightarrow\left(B,\left.\beta\right|_{B}\right), \quad \sigma(h, g)=\left(\lambda\left(h_{1}\right) \lambda\left(g_{1}\right)\right) \lambda^{-1}\left(h_{2} g_{2}\right),
$$

with convolution inverse

$$
\sigma^{-1}:(H \otimes H, \alpha \otimes \alpha) \rightarrow\left(B,\left.\beta\right|_{B}\right), \quad \sigma^{-1}(h, g)=\lambda\left(h_{1} g_{1}\right)\left(\lambda^{-1}\left(g_{2}\right) \lambda^{-1}\left(h_{2}\right)\right),
$$

where $b \in B, h, g \in H$.

Moreover, the corresponding isomorphism can be defined by

$$
\varphi:\left(B \#_{\sigma} H,\left.\beta\right|_{B} \#_{\sigma} \alpha\right) \rightarrow(A, \beta), \quad \varphi\left(b \#_{\sigma} h\right)=b \lambda(h),
$$

for all $b \in B$ and $h \in H$.

When $(H, \alpha)$ is finite dimensional, the Maschke-type theorem for Homcrossed products can be obtained by using Theorem 3.4. To prove this, the following two technical lemmas will play a great role.

Lemma 3.5. Let $\left(A \#_{\sigma} H, \beta \#_{\sigma} \alpha\right)$ be a Hom-crossed product with convolution invertible $\sigma$ and $(H, \alpha)$ a finite dimensional monoidal Hom-Hopf algebra, and let $t$ be a right integral in $(H, \alpha)$. Let $(M, \mu),(N, \nu)$ be two left $\left(A \#_{\sigma} H, \beta \#_{\sigma} \alpha\right)$-Hom-modules. If $f:(M, \mu) \rightarrow(N, \nu)$ is a left $(A, \beta)$-Hommodule morphism, then the map $\widetilde{f}:(M, \mu) \rightarrow(N, \nu)$ defined by

$$
\widetilde{f}(m)=\lambda^{-1}\left(\alpha\left(t_{1}\right)\right) \cdot f\left(\lambda\left(t_{2}\right) \cdot \mu^{-2}(m)\right),
$$

is an $\left(A \#_{\sigma} H, \beta \#_{\sigma} \alpha\right)$-Hom-module morphism, where $\lambda$ is given in Lemma 3.3.

Proof. Note first that $\alpha$-invariance of $t$ makes that $\widetilde{f}$ is a map in $\widetilde{\mathcal{H}}\left(\mathcal{M}_{k}\right)$ and that

$$
\widetilde{f}(m)=\lambda^{-1}\left(t_{1}\right) \cdot f\left(\lambda\left(\alpha^{-1}\left(t_{2}\right)\right) \cdot \mu^{-2}(m)\right) .
$$

In what follows, we will regard $(A, \beta)$ as embedded in $\left(A \#_{\sigma} H, \beta \#_{\sigma} \alpha\right)$ and write $a$ in place of $a \#{ }_{\sigma} 1_{H}, a \in A$. Since

$$
a \#{ }_{\sigma} h=\beta^{-1}(a) \lambda(h), \quad \forall a \in A, h \in H,
$$


it is sufficient to show that $\widetilde{f}$ is a morphism of $(A, \beta)$-Hom-modules, and that

$$
\widetilde{f}(\lambda(h) \cdot m)=\lambda(h) \cdot \widetilde{f}(m), \quad \forall h \in H, m \in M .
$$

Now since for all $a \in A, h \in H$,

$$
\begin{aligned}
\lambda(h) a=h_{1} \cdot & a \#_{\sigma} \alpha\left(h_{2}\right) \stackrel{(3.9)}{=} \beta^{-1}\left(h_{1} \cdot a\right) \lambda\left(\alpha\left(h_{2}\right)\right), \\
\left(\lambda\left(h_{1}\right) a\right) \lambda^{-1}\left(\alpha\left(h_{2}\right)\right) & \stackrel{(3.11)}{=}\left(\beta^{-1}\left(h_{11} \cdot a\right) \lambda\left(\alpha\left(h_{12}\right)\right)\right) \lambda^{-1}\left(\alpha\left(h_{2}\right)\right) \\
& \stackrel{(2.1)}{=}\left(h_{11} \cdot a\right)\left(\lambda\left(\alpha\left(h_{12}\right)\right) \lambda^{-1}\left(h_{2}\right)\right) \\
& \stackrel{(2.3)}{=}\left(\alpha^{-1}\left(h_{1}\right) \cdot a\right)\left(\lambda\left(\alpha\left(h_{21}\right)\right) \lambda^{-1}\left(\alpha\left(h_{22}\right)\right)\right) \\
& =\left(\alpha^{-1}\left(h_{1}\right) \cdot a\right) 1_{A} \varepsilon\left(h_{2}\right) \\
& \stackrel{(2.2)(2.4)}{=} \alpha^{-1}(h) \cdot \beta(a) .
\end{aligned}
$$

Thus we get that

$$
h \cdot a=\left(\lambda\left(\alpha\left(h_{1}\right)\right) \beta^{-1}(a)\right) \lambda^{-1}\left(\alpha^{2}\left(h_{2}\right)\right) .
$$

For all $a \in A$ and $m \in M$, we compute that

$$
\begin{aligned}
& \widetilde{f}(a \cdot m)= \lambda^{-1}\left(\alpha\left(t_{1}\right)\right) \cdot f\left(\lambda\left(t_{2}\right) \cdot\left(\beta^{-2}(a) \cdot \mu^{-2}(m)\right)\right) \\
&= \lambda^{-1}\left(\alpha\left(t_{1}\right)\right) \cdot f\left(\left(\lambda\left(\alpha^{-1}\left(t_{2}\right)\right) \beta^{-2}(a)\right) \cdot \mu^{-1}(m)\right) \\
& \stackrel{(3.11)}{=} \lambda^{-1}\left(\alpha\left(t_{1}\right)\right) \cdot f\left(\left(\left(\alpha^{-2}\left(t_{21}\right) \cdot \beta^{-3}(a)\right) \lambda\left(t_{22}\right)\right) \cdot \mu^{-1}(m)\right) \\
&= \lambda^{-1}\left(\alpha\left(t_{1}\right)\right) \cdot f\left(\left(\alpha^{-1}\left(t_{21}\right) \cdot \beta^{-2}(a)\right) \cdot\left(\lambda\left(t_{22}\right) \cdot \mu^{-2}(m)\right)\right) \\
&= \lambda^{-1}\left(\alpha\left(t_{1}\right)\right) \cdot\left[\left(\alpha^{-1}\left(t_{21}\right) \cdot \beta^{-2}(a)\right) \cdot f\left(\lambda\left(t_{22}\right) \cdot \mu^{-2}(m)\right)\right] \\
&= {\left[\lambda^{-1}\left(t_{1}\right)\left(\alpha^{-1}\left(t_{21}\right) \cdot \beta^{-2}(a)\right)\right] \cdot f\left(\lambda\left(\alpha\left(t_{22}\right)\right) \cdot \mu^{-1}(m)\right) } \\
& \stackrel{(3.12)}{=}\left\{\lambda^{-1}\left(t_{1}\right)\left[\left(\lambda\left(t_{211}\right) \beta^{-3}(a)\right) \lambda^{-1}\left(\alpha\left(t_{212}\right)\right)\right]\right\} \\
& \\
& \cdot f\left(\lambda\left(\alpha\left(t_{22}\right)\right) \cdot \mu^{-1}(m)\right) \\
&=\left\{\left[\lambda^{-1}\left(\alpha^{-1}\left(t_{1}\right)\right)\left(\lambda\left(t_{211}\right) \beta^{-3}(a)\right)\right] \lambda^{-1}\left(\alpha^{2}\left(t_{212}\right)\right)\right\} \\
& \cdot f\left(\lambda\left(\alpha\left(t_{22}\right)\right) \cdot \mu^{-1}(m)\right) \\
&=\left\{\left[\left(\lambda^{-1}\left(\alpha^{-2}\left(t_{1}\right)\right) \lambda\left(t_{\underline{111}}\right)\right) \beta^{-2}(a)\right] \lambda^{-1}\left(\alpha^{2}\left(t_{\underline{12} 2}\right)\right)\right\} \\
& \cdot f\left(\lambda\left(\alpha\left(t_{22}\right)\right) \cdot \mu^{-1}(m)\right) \\
&=\left\{\left[\left(\lambda^{-1}\left(\alpha^{-1}\left(t_{1 \underline{1}}\right)\right) \lambda\left(t_{1 \underline{12}}\right)\right) \beta^{-2}(a)\right] \lambda^{-1}\left(\alpha^{2}\left(t_{1 \underline{22}}\right)\right)\right\} \\
& \cdot f\left(\lambda\left(t_{2}\right) \cdot \mu^{-1}(m)\right)
\end{aligned}
$$


EQUIVALENT CROSSED PRODUCTS OF MONOIDAL HOM-HOPF ALGEBRAS 353

$$
\begin{aligned}
& =\left\{\left[\left(\lambda^{-1}\left(t_{111}\right) \lambda\left(t_{112}\right)\right) \beta^{-2}(a)\right] \lambda^{-1}\left(\alpha\left(t_{12}\right)\right)\right\} \cdot f\left(\lambda\left(t_{2}\right) \cdot \mu^{-1}(m)\right) \\
& =\left(\beta^{-1}(a) \lambda^{-1}\left(t_{1}\right)\right) \cdot f\left(\lambda\left(t_{2}\right) \cdot \mu^{-1}(m)\right) \\
& =a \cdot\left[\lambda^{-1}\left(t_{1}\right) \cdot f\left(\lambda\left(\alpha^{-1}\left(t_{2}\right)\right) \cdot \mu^{-2}(m)\right)\right] \\
& =a \cdot \widetilde{f}(m),
\end{aligned}
$$

which implies that $\tilde{f}$ is a morphism of $(A, \beta)$-Hom-modules.

Last, to show that (3.10) holds, we need the formula:

$$
\sigma(h, g)=\left(\lambda\left(\alpha\left(h_{1}\right)\right) \lambda\left(\alpha\left(g_{1}\right)\right)\right) \lambda^{-1}\left(\alpha\left(h_{2} g_{2}\right)\right),
$$

for all $h, g \in H$, which can be proved by the following: since

$$
\begin{aligned}
\lambda(h) \lambda(g) & =\left(1_{A} \#_{\sigma} \alpha^{-1}(h)\right)\left(1_{A} \#_{\sigma} \alpha^{-1}(g)\right) \\
& =1_{A}\left(\left(\alpha^{-2}\left(h_{1}\right) \cdot 1_{A}\right) \sigma\left(\alpha^{-1}\left(h_{21}\right), \alpha^{-2}\left(g_{1}\right)\right)\right) \#_{\sigma} \alpha\left(h_{22}\right) g_{2} \\
& =1_{A}\left(\left(\alpha^{-1}\left(h_{11}\right) \cdot 1_{A}\right) \sigma\left(\alpha^{-1}\left(h_{12}\right), \alpha^{-2}\left(g_{1}\right)\right)\right) \#_{\sigma} h_{2} g_{2} \\
& =\sigma\left(h_{1}, g_{1}\right) \#_{\sigma} h_{2} g_{2} \stackrel{(3.9)}{=} \sigma\left(\alpha^{-1}\left(h_{1}\right), \alpha^{-1}\left(g_{1}\right)\right) \lambda\left(h_{2} g_{2}\right),
\end{aligned}
$$

we obtain that

$$
\begin{aligned}
& \left(\lambda\left(\alpha\left(h_{1}\right)\right) \lambda\left(\alpha\left(g_{1}\right)\right)\right) \lambda^{-1}\left(\alpha\left(h_{2} g_{2}\right)\right) \\
& \quad=\left(\sigma\left(h_{11}, g_{11}\right) \lambda\left(\alpha\left(h_{12} g_{12}\right)\right)\right) \lambda^{-1}\left(\alpha\left(h_{2} g_{2}\right)\right) \\
& \quad=\sigma\left(\alpha\left(h_{11}\right), \alpha\left(g_{11}\right)\right)\left(\lambda\left(\alpha\left(h_{12} g_{12}\right)\right) \lambda^{-1}\left(h_{2} g_{2}\right)\right) \\
& \quad=\sigma\left(h_{1}, g_{1}\right)\left(\lambda\left(\alpha\left(h_{21} g_{21}\right)\right) \lambda^{-1}\left(\alpha\left(h_{22} g_{22}\right)\right)\right) \\
& \quad=\sigma(h, g) .
\end{aligned}
$$

We now have that

$$
\begin{aligned}
\widetilde{f} & (\lambda(h) \cdot m) \\
& =\lambda^{-1}\left(\alpha\left(t_{1}\right)\right) \cdot f\left(\lambda\left(t_{2}\right) \cdot\left(\lambda\left(\alpha^{-2}(h)\right) \cdot \mu^{-2}(m)\right)\right) \\
& \stackrel{(2.8)}{=} \lambda^{-1}\left(\alpha\left(t_{1}\right)\right) \cdot f\left(\left(\lambda\left(\alpha^{-1}\left(t_{2}\right)\right) \lambda\left(\alpha^{-2}(h)\right)\right) \cdot \mu^{-1}(m)\right) \\
& =\lambda^{-1}\left(\alpha\left(t_{1}\right)\right) \cdot f\left(\left(\sigma\left(\alpha^{-2}\left(t_{21}\right), \alpha^{-3}\left(h_{1}\right)\right) \lambda\left(\alpha^{-1}\left(t_{22}\right) \alpha^{-2}\left(h_{2}\right)\right)\right) \cdot \mu^{-1}(m)\right) \\
& =\lambda^{-1}\left(\alpha\left(t_{1}\right)\right) \cdot f\left(\sigma\left(\alpha^{-1}\left(t_{21}\right), \alpha^{-2}\left(h_{1}\right)\right) \cdot\left(\lambda\left(\alpha^{-1}\left(t_{22}\right) \alpha^{-2}\left(h_{2}\right)\right) \cdot \mu^{-2}(m)\right)\right) \\
& =\lambda^{-1}\left(\alpha\left(t_{1}\right)\right) \cdot\left[\sigma\left(\alpha^{-1}\left(t_{21}\right), \alpha^{-2}\left(h_{1}\right)\right) \cdot f\left(\lambda\left(\alpha^{-1}\left(t_{22}\right) \alpha^{-2}\left(h_{2}\right)\right) \cdot \mu^{-2}(m)\right)\right] \\
& =\left(\lambda^{-1}\left(t_{1}\right) \sigma\left(\alpha^{-1}\left(t_{21}\right), \alpha^{-2}\left(h_{1}\right)\right)\right) \cdot f\left(\lambda\left(t_{22} \alpha^{-1}\left(h_{2}\right)\right) \cdot \mu^{-1}(m)\right) \\
& =\left(\lambda^{-1}\left(\alpha\left(t_{11}\right)\right) \sigma\left(\alpha^{-1}\left(t_{12}\right), \alpha^{-2}\left(h_{1}\right)\right)\right) \cdot f\left(\lambda\left(\alpha^{-1}\left(t_{2} h_{2}\right)\right) \cdot \mu^{-1}(m)\right) \\
& \stackrel{(3.13)}{=}\left\{\lambda^{-1}\left(\alpha\left(t_{11}\right)\right)\left[\left(\lambda\left(t_{121}\right) \lambda\left(\alpha^{-1}\left(h_{11}\right)\right)\right) \lambda^{-1}\left(t_{122} \alpha^{-1}\left(h_{12}\right)\right)\right]\right\}
\end{aligned}
$$




$$
\begin{aligned}
& \cdot f\left(\lambda\left(\alpha^{-1}\left(t_{2} h_{2}\right)\right) \cdot \mu^{-1}(m)\right) \\
& =\left\{\left[\lambda^{-1}\left(t_{11}\right)\left(\lambda\left(t_{121}\right) \lambda\left(\alpha^{-1}\left(h_{11}\right)\right)\right)\right] \lambda^{-1}\left(\alpha\left(t_{122}\right) h_{12}\right)\right\} \\
& \cdot f\left(\lambda\left(\alpha^{-1}\left(t_{2} h_{2}\right)\right) \cdot \mu^{-1}(m)\right) \\
& =\left\{\left[\left(\lambda^{-1}\left(\alpha^{-1}\left(t_{1 \underline{1}}\right)\right) \lambda\left(t_{1 \underline{21}}\right)\right) \lambda\left(h_{11}\right)\right] \lambda^{-1}\left(\alpha\left(t_{1 \underline{22}}\right) h_{12}\right)\right\} \\
& \cdot f\left(\lambda\left(\alpha^{-1}\left(t_{2} h_{2}\right)\right) \cdot \mu^{-1}(m)\right) \\
& =\left\{\left[\left(\lambda^{-1}\left(t_{111}\right) \lambda\left(t_{112}\right)\right) \lambda\left(h_{11}\right)\right] \lambda^{-1}\left(t_{12} h_{12}\right)\right\} \\
& \cdot f\left(\lambda\left(\alpha^{-1}\left(t_{2} h_{2}\right)\right) \cdot \mu^{-1}(m)\right) \\
& =\left(\lambda\left(\alpha\left(h_{11}\right)\right) \lambda^{-1}\left(\alpha^{-1}\left(t_{1}\right) h_{12}\right)\right) \cdot f\left(\lambda\left(\alpha^{-1}\left(t_{2} h_{2}\right)\right) \cdot \mu^{-1}(m)\right) \\
& =\left(\lambda\left(h_{1}\right) \lambda^{-1}\left(t_{1} h_{21}\right)\right) \cdot f\left(\lambda\left(t_{2} h_{22}\right) \cdot \mu^{-1}(m)\right) \\
& \stackrel{(3.14)}{=}\left(\lambda\left(\alpha^{-1}(h)\right) \lambda^{-1}\left(t_{1}\right)\right) \cdot f\left(\lambda\left(t_{2}\right) \cdot \mu^{-1}(m)\right) \\
& =\lambda(h) \cdot\left[\lambda^{-1}\left(t_{1}\right) \cdot f\left(\lambda\left(\alpha^{-1}\left(t_{2}\right)\right) \cdot \mu^{-2}(m)\right)\right] \\
& =\lambda(h) \cdot \widetilde{f}(m) \text {, }
\end{aligned}
$$

as required, where (3.14) is given by the following

$$
\begin{aligned}
h_{1} \otimes\left(t_{1} h_{21} \otimes t_{2} h_{22}\right) & =h_{1} \otimes\left(\left(t h_{2}\right)_{1} \otimes\left(t h_{2}\right)_{2}\right)=\varepsilon\left(h_{2}\right) h_{1} \otimes\left(t_{1} \otimes t_{2}\right) \\
& =\alpha^{-1}(h) \otimes \Delta(t) .
\end{aligned}
$$

Hence, the proof of this lemma is completed.

Lemma 3.6. Let $\left(A \#_{\sigma} H, \beta \#_{\sigma} \alpha\right)$ be a Hom-crossed product with convolution invertible $\sigma$ and $(H, \alpha)$ a finite dimensional semisimple monoidal HomHopf algebra. Let $(M, \mu)$ be a left $\left(A \#_{\sigma} H, \beta \#_{\sigma} \alpha\right)$-Hom-module and $(N, \nu)$ an $\left(A \#_{\sigma} H, \beta \#_{\sigma} \alpha\right)$-Hom-submodule of $(M, \mu)$. If $(N, \nu)$ is a direct summand of $(M, \mu)$ as $(A, \beta)$-Hom-modules, then, $(N, \nu)$ is also a direct summand of $(M, \mu)$ as $\left(A \#_{\sigma} H, \beta \#_{\sigma} \alpha\right)$-Hom-modules.

Proof. Let $\pi:(M, \mu) \rightarrow(N, \nu)$ be the canonical projection as $(A, \beta)$ Hom-modules. By the assumption of $(H, \alpha)$ and [6, Theorem 3.6], there exists a normalized right integral $t$. Then the map

$$
\tilde{\pi}:(M, \mu) \rightarrow(N, \nu), \quad \tilde{\pi}(m)=\lambda^{-1}\left(\alpha\left(t_{1}\right)\right) \cdot \pi\left(\lambda\left(t_{2}\right) \cdot \mu^{-2}(m)\right),
$$

is an $\left(A \#_{\sigma} H, \beta \#_{\sigma} \alpha\right)$-Hom-module morphism by Lemma 3.5. Furthermore, $\widetilde{\pi}$ is also a projection. 
EQUIVALENT CROSSED PRODUCTS OF MONOIDAL HOM-HOPF ALGEBRAS 355

Indeed, for all $n \in M$, the projectivity of $\pi$ implies that

$$
\begin{aligned}
\widetilde{\pi}(n) & =\lambda^{-1}\left(\alpha\left(t_{1}\right)\right) \cdot\left(\lambda\left(t_{2}\right) \cdot \mu^{-2}(n)\right) \\
& =\left(\lambda^{-1}\left(t_{1}\right) \lambda\left(t_{2}\right)\right) \cdot \mu^{-1}(n) \\
& =\varepsilon(t) 1_{A} \cdot \mu^{-1}(n) \\
& =n,
\end{aligned}
$$

which completes the proof of this lemma.

Combining above two lemmas, we obtain the following Maschke-type theorem for Hom-crossed products.

THEOREM 3.7. Let $\left(A \#_{\sigma} H, \beta \#_{\sigma} \alpha\right)$ be a Hom-crossed product with convolution invertible $\sigma$ and $(H, \alpha)$ a finite dimensional semisimple monoidal Hom-Hopf algebra. If $(A, \beta)$ is semisimple, then so is $\left(A \#_{\sigma} H, \beta \#_{\sigma} \alpha\right)$.

\section{Equivalent Hom-Crossed PRoducts}

In this section, we introduce the conception of equivalent Hom-crossed systems and investigate the sufficient and necessary conditions for two Homcrossed products to be equivalent, meanwhile, we construct an equivalent Hom-crossed system based on a same Hom-crossed product, by using lazy Hom-2-cocyles.

Proposition 4.1. Let $\left(A \#_{\sigma} H, \beta \# \alpha\right)$ be a Hom-crossed product such that the weak action of $(H, \alpha)$ on $(A, \beta)$ is inner via some convolution invertible map $\lambda:(H, \alpha) \rightarrow(A, \beta)$ in $\widetilde{\mathcal{H}}\left(\mathcal{M}_{k}\right)$. Define

$$
\begin{aligned}
& \tau:(H \otimes H, \alpha \otimes \alpha) \rightarrow(A, \beta), \\
& \tau(h, g)=\left[\left(\left(\lambda^{-1}\left(g_{11}\right) \lambda^{-1}\left(h_{11}\right)\right) \sigma\left(h_{12}, g_{12}\right)\right] \lambda\left(h_{2} g_{2}\right) .\right.
\end{aligned}
$$

Then $\tau$ is a Hom-cocycle such that

$$
\left(A \#_{\sigma} H, \beta \#_{\sigma} \alpha\right) \cong\left(A_{\tau}[H], \beta \otimes_{\tau} \alpha\right),
$$

via a Hom-algebra morphism which is also left $(A, \beta)$-linear, right $(H, \alpha)$ colinear morphism.

Proof. For all $a \in A$ and $h \in H$, define

$$
\phi: A \#_{\sigma} H \rightarrow A_{\tau}[H], \quad \phi\left(a \#_{\sigma} h\right)=\beta^{-1}(a) \lambda\left(h_{1}\right) \otimes_{\tau} \alpha\left(h_{2}\right) .
$$

Then we claim that $\phi$ is bijective with inverse

$$
\psi: A_{\tau}[H] \rightarrow A_{\sigma} H, \psi\left(a \otimes_{\tau} h\right)=\beta^{-1}(a) \lambda^{-1}\left(h_{1}\right) \#_{\sigma} \alpha\left(h_{2}\right) .
$$


Indeed, on one hand,

$$
\begin{aligned}
(\phi \circ \psi)\left(a \otimes_{\tau} h\right) & =\beta^{-1}\left(\beta^{-1}(a) \lambda^{-1}\left(h_{1}\right)\right) \lambda\left(\alpha\left(h_{21}\right)\right) \otimes_{\tau} \alpha^{2}\left(h_{22}\right) \\
& =\left(\beta^{-2}(a) \lambda^{-1}\left(\alpha^{-1}\left(h_{1}\right)\right)\right) \lambda\left(\alpha\left(h_{21}\right)\right) \otimes_{\tau} \alpha^{2}\left(h_{22}\right) \\
& =\left(\beta^{-2}(a) \lambda^{-1}\left(h_{11}\right)\right) \lambda\left(\alpha\left(h_{12}\right)\right) \otimes_{\tau} \alpha\left(h_{2}\right) \\
& =\beta^{-1}(a)\left(\lambda^{-1}\left(h_{11}\right) \lambda\left(h_{12}\right)\right) \otimes_{\tau} \alpha\left(h_{2}\right) \\
& =\beta^{-1}(a) 1_{A} \otimes_{\tau} \varepsilon\left(h_{1}\right) \alpha\left(h_{2}\right) \\
& =a \otimes_{\tau} h .
\end{aligned}
$$

On the other hand, $\psi \circ \phi=i d$ can be similarly proved.

We now check that $\phi$ is a morphism of monoidal Hom-algebras: the fact that $\phi$ preserves units is obvious, and for all $a, b \in A, h, g \in H$,

$$
\begin{aligned}
& \phi\left(\left(a \#_{\sigma} h\right)\left(b \#{ }_{\sigma} g\right)\right) \\
& =\phi\left[a\left(\left(\alpha^{-1}\left(h_{1}\right) \cdot \beta^{-2}(b)\right) \sigma\left(h_{21}, \alpha^{-1}\left(g_{1}\right)\right)\right) \#_{\sigma} \alpha^{2}\left(h_{22}\right) \alpha\left(g_{2}\right)\right] \\
& =\left\{\beta^{-1}(a)\left[\left(\alpha^{-2}\left(h_{1}\right) \cdot \beta^{-3}(b)\right) \sigma\left(\alpha^{-1}\left(h_{21}\right), \alpha^{-2}\left(g_{1}\right)\right)\right]\right\} \\
& \lambda\left(\alpha^{2}\left(h_{221}\right) \alpha\left(g_{21}\right)\right) \otimes_{\tau} \alpha^{3}\left(h_{222}\right) \alpha^{2}\left(g_{22}\right) \\
& =\left\{\left[\beta^{-2}(a)\left(\alpha^{-2}\left(h_{1}\right) \cdot \beta^{-3}(b)\right)\right] \sigma\left(h_{21}, \alpha^{-1}\left(g_{1}\right)\right)\right\} \\
& \lambda\left(\alpha^{2}\left(h_{221}\right) \alpha\left(g_{21}\right)\right) \otimes_{\tau} \alpha^{3}\left(h_{222}\right) \alpha^{2}\left(g_{22}\right) \\
& =\left[\beta^{-1}(a)\left(\alpha^{-1}\left(h_{1}\right) \cdot \beta^{-2}(b)\right)\right]\left(\sigma\left(h_{21}, \alpha^{-1}\left(g_{1}\right)\right) \lambda\left(\alpha\left(h_{221}\right) g_{21}\right)\right) \\
& \otimes_{\tau} \alpha^{3}\left(h_{222}\right) \alpha^{2}\left(g_{22}\right) \\
& \stackrel{(3.4)}{=}\left\{\beta^{-1}(a)\left[\left(\lambda\left(\alpha^{-1}\left(h_{11}\right)\right) \beta^{-3}(b)\right) \lambda^{-1}\left(h_{12}\right)\right]\right\} \\
& \times\left(\sigma\left(h_{21}, \alpha^{-1}\left(g_{1}\right)\right) \lambda\left(\alpha\left(h_{221}\right) g_{21}\right)\right) \otimes_{\tau} \alpha^{3}\left(h_{222}\right) \alpha^{2}\left(g_{22}\right) \\
& =\left\{\left[\beta^{-2}(a)\left(\lambda\left(\alpha^{-1}\left(h_{11}\right)\right) \beta^{-3}(b)\right)\right] \lambda^{-1}\left(\alpha\left(h_{12}\right)\right)\right\} \\
& \times\left(\sigma\left(h_{21}, \alpha^{-1}\left(g_{1}\right)\right) \lambda\left(\alpha\left(h_{221}\right) g_{21}\right)\right) \otimes_{\tau} \alpha^{3}\left(h_{222}\right) \alpha^{2}\left(g_{22}\right) \\
& =\left[\beta^{-1}(a)\left(\lambda\left(h_{11}\right) \beta^{-2}(b)\right)\right] \\
& \times\left[\lambda^{-1}\left(\alpha\left(h_{12}\right)\right)\left(\sigma\left(\alpha^{-1}\left(h_{21}\right), \alpha^{-2}\left(g_{1}\right)\right) \lambda\left(h_{221} \alpha^{-1}\left(g_{21}\right)\right)\right)\right] \\
& \otimes_{\tau} \alpha^{3}\left(h_{222}\right) \alpha^{2}\left(g_{22}\right) \\
& =\left[\left(\beta^{-2}(a) \lambda\left(h_{11}\right)\right) \beta^{-1}(b)\right] \\
& \times\left[\left(\lambda^{-1}\left(h_{12}\right) \sigma\left(\alpha^{-1}\left(h_{21}\right), \alpha^{-2}\left(g_{1}\right)\right)\right) \lambda\left(\alpha\left(h_{221}\right) g_{21}\right)\right] \otimes_{\tau} \alpha^{3}\left(h_{222}\right) \alpha^{2}\left(g_{22}\right) \\
& =\left[\left(\beta^{-2}(a) \lambda\left(h_{11}\right)\right)\left(\beta^{-2}(b) 1_{A} \varepsilon\left(g_{11}\right)\right)\right] \\
& \times\left[\left(\lambda^{-1}\left(h_{12}\right) \sigma\left(\alpha^{-1}\left(h_{21}\right), \alpha^{-1}\left(g_{12}\right)\right)\right) \lambda\left(\alpha\left(h_{221}\right) g_{21}\right)\right] \otimes_{\tau} \alpha^{3}\left(h_{222}\right) \alpha^{2}\left(g_{22}\right) \\
& =\left\{\left(\beta^{-2}(a) \lambda\left(h_{11}\right)\right)\left[\beta^{-2}(b)\left(\lambda\left(g_{111}\right) \lambda^{-1}\left(g_{112}\right)\right)\right]\right\} \\
& \times\left[\left(\lambda^{-1}\left(h_{12}\right) \sigma\left(\alpha^{-1}\left(h_{21}\right), \alpha^{-1}\left(g_{12}\right)\right)\right) \lambda\left(\alpha\left(h_{221}\right) g_{21}\right)\right] \otimes_{\tau} \alpha^{3}\left(h_{222}\right) \alpha^{2}\left(g_{22}\right)
\end{aligned}
$$


EQUIVALENT CROSSED PRODUCTS OF MONOIDAL HOM-HOPF ALGEBRAS 357

$$
\begin{aligned}
& =\left\{\left(\beta^{-2}(a) \lambda\left(h_{11}\right)\right)\left[\left(\beta^{-3}(b) \lambda\left(g_{111}\right)\right) \lambda^{-1}\left(\alpha\left(g_{112}\right)\right)\right]\right\} \\
& \times\left[\left(\lambda^{-1}\left(h_{12}\right) \sigma\left(\alpha^{-1}\left(h_{21}\right), \alpha^{-1}\left(g_{12}\right)\right)\right) \lambda\left(\alpha\left(h_{221}\right) g_{21}\right)\right] \otimes_{\tau} \alpha^{3}\left(h_{222}\right) \alpha^{2}\left(g_{22}\right) \\
& =\left\{\left[\left(\beta^{-3}(a) \lambda\left(\alpha^{-1}\left(h_{11}\right)\right)\right)\left(\beta^{-3}(b) \lambda\left(g_{111}\right)\right)\right] \lambda^{-1}\left(\alpha^{2}\left(g_{112}\right)\right)\right\} \\
& \times\left[\left(\lambda^{-1}\left(h_{12}\right) \sigma\left(\alpha^{-1}\left(h_{21}\right), \alpha^{-1}\left(g_{12}\right)\right)\right) \lambda\left(\alpha\left(h_{221}\right) g_{21}\right)\right] \otimes_{\tau} \alpha^{3}\left(h_{222}\right) \alpha^{2}\left(g_{22}\right) \\
& =\left[\left(\beta^{-2}(a) \lambda\left(h_{11}\right)\right)\left(\beta^{-2}(b) \lambda\left(\alpha\left(g_{111}\right)\right)\right)\right] \\
& \times\left\{\lambda ^ { - 1 } ( \alpha ^ { 2 } ( g _ { 1 1 2 } ) ) \left[\left(\lambda^{-1}\left(\alpha^{-1}\left(h_{12}\right)\right) \sigma\left(\alpha^{-2}\left(h_{21}\right), \alpha^{-2}\left(g_{12}\right)\right)\right)\right.\right. \\
& \left.\left.\lambda\left(h_{221} \alpha^{-1}\left(g_{21}\right)\right)\right]\right\} \otimes_{\tau} \alpha^{3}\left(h_{222}\right) \alpha^{2}\left(g_{22}\right) \\
& =\left[\left(\beta^{-2}(a) \lambda\left(h_{11}\right)\right)\left(\beta^{-2}(b) \lambda\left(\alpha\left(g_{111}\right)\right)\right)\right] \\
& \times\left\{\left[\lambda^{-1}\left(\alpha\left(g_{112}\right)\right)\left(\lambda^{-1}\left(\alpha^{-1}\left(h_{12}\right)\right) \sigma\left(\alpha^{-2}\left(h_{21}\right), \alpha^{-2}\left(g_{12}\right)\right)\right)\right]\right. \\
& \left.\lambda\left(\alpha\left(h_{221}\right) g_{21}\right)\right\} \otimes_{\tau} \alpha^{3}\left(h_{222}\right) \alpha^{2}\left(g_{22}\right) \\
& =\left[\left(\beta^{-2}(a) \lambda\left(h_{\underline{11}}\right)\right)\left(\beta^{-2}(b) \lambda\left(\alpha\left(g_{\underline{111}}\right)\right)\right)\right] \\
& \times\left\{\left[\left(\lambda^{-1}\left(g_{1 \underline{\underline{12}}}\right) \lambda^{-1}\left(\alpha^{-1}\left(h_{\underline{12}}\right)\right)\right) \sigma\left(\alpha^{-1}\left(h_{\underline{21}}\right), \alpha^{-1}\left(g_{1 \underline{\underline{2}}}\right)\right)\right] \lambda\left(\alpha\left(h_{\underline{2} 21}\right) g_{21}\right)\right\} \\
& \otimes_{\tau} \alpha^{3}\left(h_{222}\right) \alpha^{2}\left(g_{22}\right) \\
& =\left[\left(\beta^{-2}(a) \lambda\left(\alpha^{-1}\left(h_{1}\right)\right)\right)\left(\beta^{-2}(b) \lambda\left(g_{\underline{\underline{11}}}\right)\right)\right] \\
& \times\left\{\left[\left(\lambda^{-1}\left(g_{\underline{\underline{121}}}\right) \lambda^{-1}\left(\alpha^{-1}\left(h_{21}\right)\right)\right) \sigma\left(h_{22 \underline{1}}, g_{\underline{\underline{122}}}\right)\right] \lambda\left(\alpha^{2}\left(h_{22 \underline{21}}\right) g_{\underline{\underline{21}}}\right)\right\} \\
& \otimes_{\tau} \alpha^{4}\left(h_{22 \underline{22}}\right) \alpha^{2}\left(g_{\underline{\underline{2}} 2}\right) \\
& =\left[\left(\beta^{-2}(a) \lambda\left(\alpha^{-1}\left(h_{1}\right)\right)\right)\left(\beta^{-2}(b) \lambda\left(\alpha^{-1}\left(g_{1}\right)\right)\right)\right] \\
& \times\left\{\left[\left(\lambda^{-1}\left(g_{2 \underline{\underline{11}}}\right) \lambda^{-1}\left(\alpha^{-1}\left(h_{2 \underline{1}}\right)\right)\right) \sigma\left(\alpha\left(h_{2 \underline{211}}\right), g_{2 \underline{11}}\right)\right] \lambda\left(\alpha^{2}\left(h_{2 \underline{212}}\right) \alpha\left(g_{2 \underline{21}}\right)\right)\right\} \\
& \otimes_{\tau} \alpha^{3}\left(h_{2 \underline{22}} g_{2 \underline{2}}\right) \\
& =\left[\left(\beta^{-2}(a) \lambda\left(\alpha^{-1}\left(h_{1}\right)\right)\right)\left(\beta^{-2}(b) \lambda\left(\alpha^{-1}\left(g_{1}\right)\right)\right)\right] \\
& \times\left\{\left[\left(\lambda^{-1}\left(\alpha\left(g_{2111}\right)\right) \lambda^{-1}\left(h_{21 \underline{1}}\right)\right) \sigma\left(\alpha\left(h_{2121}\right), \alpha\left(g_{2112}\right)\right)\right] \lambda\left(\alpha^{2}\left(h_{2122}\right) \alpha\left(g_{212}\right)\right)\right\} \\
& \otimes_{\tau} \alpha^{2}\left(h_{22} g_{22}\right) \\
& =\left[\left(\beta^{-2}(a) \lambda\left(\alpha^{-1}\left(h_{1}\right)\right)\right)\left(\beta^{-2}(b) \lambda\left(\alpha^{-1}\left(g_{1}\right)\right)\right)\right] \\
& \times\left\{\left[\left(\lambda^{-1}\left(\alpha\left(g_{2111}\right)\right) \lambda^{-1}\left(\alpha\left(h_{2111}\right)\right)\right) \sigma\left(\alpha\left(h_{2112}\right), \alpha\left(g_{2112}\right)\right)\right] \lambda\left(\alpha\left(h_{212} g_{212}\right)\right)\right\} \\
& \otimes_{\tau} \alpha^{2}\left(h_{22} g_{22}\right) \\
& \stackrel{(3.7)}{=}\left(\beta^{-1}(a) \lambda\left(h_{1}\right) \otimes_{\tau} \alpha\left(h_{2}\right)\right)\left(\beta^{-1}(b) \lambda\left(g_{1}\right) \otimes_{\tau} \alpha\left(g_{2}\right)\right) \\
& =\phi\left(a \#_{\sigma} h\right) \phi\left(b \#_{\sigma} g\right) \text {. }
\end{aligned}
$$

Since $\left(A \#_{\sigma} H, \beta \#_{\sigma} \alpha\right) \cong\left(A_{\tau}[H], \beta \otimes_{\tau} \alpha\right)$ as monoidal Hom-algebras, $A_{\tau}[H]$ is Hom-associative, and thus $\tau$ is a Hom-cocycle. 
Last, it is straightforward to check that $\phi$ is a left $(A, \beta)$-Hom-module and a right $(H, \alpha)$-Hom-comodule morphism. Indeed, for all $a, b \in A$ and $g \in H$, we have that

$$
\begin{aligned}
\phi\left(a \cdot\left(b \#_{\sigma} g\right)\right) & =\phi\left(a b \#_{\sigma} \alpha(g)\right) \\
& =\beta^{-1}(a b) \lambda\left(\alpha\left(g_{1}\right)\right) \otimes_{\tau} \alpha^{2}\left(g_{2}\right) \\
& =a\left(\beta^{-1}(b) \lambda\left(g_{1}\right)\right) \otimes_{\tau} \alpha^{2}\left(g_{2}\right) \\
& =a \cdot\left(\beta^{-1}(b) \lambda\left(g_{1}\right) \otimes_{\tau} \alpha\left(g_{2}\right)\right) \\
& =a \cdot \phi\left(b \#_{\sigma} g\right),
\end{aligned}
$$

and

$$
\begin{aligned}
(\rho \circ \phi)\left(b \#_{\sigma} g\right) & =\rho\left(\beta^{-1}(b) \lambda\left(g_{1}\right) \otimes_{\tau} \alpha\left(g_{2}\right)\right) \\
& =\left(\beta^{-2}(b) \lambda\left(\alpha^{-1}\left(g_{1}\right)\right) \otimes_{\tau} \alpha\left(g_{21}\right)\right) \otimes \alpha^{2}\left(g_{22}\right) \\
& =\left(\beta^{-2}(b) \lambda\left(g_{11}\right) \otimes_{\tau} \alpha\left(g_{12}\right)\right) \otimes \alpha\left(g_{2}\right) \\
& =\phi\left(\beta^{-1}(b) \#_{\sigma} g_{1}\right) \otimes \alpha\left(g_{2}\right) \\
& =((\phi \otimes i d) \circ \rho)\left(b \#_{\sigma} g\right),
\end{aligned}
$$

as needed.

Hence, we complete the proof of this proposition.

EXAMPLE 4.2. Let $(A \# H, \beta \# \alpha)$ be a Hom-smash product such that the $H$-action is inner via some Hom-algebra morphism from $(H, \alpha)$ to $(A, \beta)$. Then $(A \# H, \beta \# \alpha) \cong(A \otimes H, \beta \otimes \alpha)$, for the cocycle $\tau$ in Proposition 4.1. It is easy to see that the cocycle $\tau$ is trivial.

The inverse of Proposition 4.1 is also true: that is, if $\left(A \#_{\sigma} H, \beta \#_{\sigma} \alpha\right) \cong$ $\left(A_{\tau}[H], \beta \otimes_{\tau} \alpha\right)$ for some Hom-twist product, by a Hom-algebra isomorphism which is a left $(A, \beta)$-Hom-module, right $(H, \alpha)$-Hom-comodule morphism, then the original Hom-action must be inner, via the convolution invertible map $\lambda:(H, \alpha) \rightarrow(A, \beta)$ in $\widetilde{\mathcal{H}}\left(\mathcal{M}_{k}\right)$ that is given in Proposition 4.1. In fact, one can more generally give a necessary and sufficient condition for two Hom-crossed products to be isomorphic, which is a generalization of [1] and [8].

TheOrem 4.3. Let $(A, \beta)$ be a monoidal Hom-algebra and $(H, \alpha)$ a monoidal Hom-Hopf algebra, with two weak actions $h \otimes a \mapsto h \cdot a, h \otimes a \mapsto h \cdot^{\prime} a$, with respect to two cocycles $\sigma, \sigma^{\prime}: H \otimes H \rightarrow A$, respectively. Assume that

$$
\phi:\left(A \#_{\sigma} H, \beta \#_{\sigma} \alpha\right) \rightarrow\left(A \#_{\sigma^{\prime}} H, \beta \#_{\sigma^{\prime}} \alpha\right)
$$

is a Hom-algebra isomorphism, which is a left $(A, \beta)$-Hom-module and right $(H, \alpha)$-Hom-comodule morphism. Then there exists an convolution invertible morphism $\lambda:(H, \alpha) \rightarrow(A, \beta)$ in $\widetilde{\mathcal{H}}\left(\mathcal{M}_{k}\right)$ such that for all $a \in A, h, g \in H$,

(1) $\phi\left(a \#_{\sigma} h\right)=\beta^{-1}(a) \lambda\left(h_{1}\right) \#_{\sigma^{\prime}} \alpha\left(h_{2}\right)$, 
EQUIVALENT CROSSED PRODUCTS OF MONOIDAL HOM-HOPF ALGEBRAS 359

(2) $h \cdot^{\prime} a=\left[\lambda^{-1}\left(\alpha\left(h_{11}\right)\right)\left(h_{12} \cdot \beta^{-2}(a)\right)\right] \lambda\left(\alpha\left(h_{2}\right)\right)$,

(3) $\sigma^{\prime}(h, g)=\left[\lambda^{-1}\left(\alpha\left(h_{11}\right)\right)\left(h_{12} \cdot \lambda^{-1}\left(\alpha^{-1}\left(g_{1}\right)\right)\right)\right]\left[\sigma\left(h_{21}, g_{21}\right) \lambda\left(h_{22} g_{22}\right)\right]$.

Conversely, given a convolution invertible map $\lambda:(H, \alpha) \rightarrow(A, \beta)$ in $\widetilde{\mathcal{H}}\left(\mathcal{M}_{k}\right)$ such that (2) and (3) hold, then the map $\phi$ in (1) is an isomorphism of Homalgebras, which is also left $(A, \beta)$-linear, right $(H, \alpha)$-colinear.

Proof. Define $\lambda:(H, \alpha) \rightarrow(A, \beta)$ by $\lambda(h)=((i d \otimes \varepsilon) \circ \phi)\left(1_{A} \#_{\sigma} h\right)$ for all $h \in H$, which is clearly a morphism in $\widetilde{\mathcal{H}}\left(\mathcal{M}_{k}\right)$.

Noticing that $(i d \otimes \varepsilon) \circ \phi$ is a morphism of left $(A, \beta)$-Hom-modules, we get that

$$
\begin{aligned}
((i d \otimes \varepsilon) \circ \phi)\left(a \#{ }_{\sigma} h\right) & =\beta^{-1}(a)((i d \otimes \varepsilon) \circ \phi)\left(1_{A} \# \sigma_{\sigma} \alpha^{-1}(h)\right) \\
& =\beta^{-1}(a) \lambda\left(\alpha^{-1}(h)\right),
\end{aligned}
$$

for all $a \in A, h \in H$. On the other hand, since $\phi$ is a morphism of right $(H, \alpha)$-Hom-comodules, we have

$$
(\rho \circ \phi)\left(a \#{ }_{\sigma} h\right)=\phi\left(\beta^{-1}(a) \#_{\sigma} h_{1}\right) \otimes \alpha\left(h_{2}\right) .
$$

Writing $\phi\left(1_{A} \#_{\sigma} \alpha^{-1}(h)\right)=\sum_{i} a_{i} \#_{\sigma^{\prime}} h_{i} \in A \#_{\sigma^{\prime}} H$ and applying $(i d \otimes \varepsilon) \otimes$ $i d$ to both sides of above equation, we obtain that

$$
\begin{aligned}
(((i d) & \otimes \varepsilon) \otimes i d) \circ \rho \circ \phi)\left(a \# \#_{\sigma} h\right) \\
& =(((i d \otimes \varepsilon) \otimes i d) \circ \rho)\left(\beta^{-1}(a) \cdot \phi\left(1_{A} \#_{\sigma} \alpha^{-1}(h)\right)\right) \\
& =(((i d \otimes \varepsilon) \otimes i d) \circ \rho)\left(\beta^{-1}(a) \cdot\left(\sum_{i} a_{i} \#_{\sigma^{\prime}} h_{i}\right)\right) \\
& =((i d \otimes \varepsilon) \otimes i d) \circ \rho)\left(\sum_{i} \beta^{-1}(a) a_{i} \#_{\sigma^{\prime}} \alpha\left(h_{i}\right)\right) \\
& =((i d \otimes \varepsilon) \otimes i d)\left(\left(\sum_{i} \beta^{-2}(a) \beta^{-1}\left(a_{i}\right) \#_{\sigma^{\prime}} \alpha\left(h_{i 1}\right)\right) \otimes \alpha^{2}\left(h_{i 2}\right)\right) \\
& =\sum_{i} \beta^{-2}(a) \beta^{-1}\left(a_{i}\right) \otimes \alpha\left(h_{i}\right), \\
((i d \otimes & \otimes \\
& (\stackrel{4}{ }) \otimes i d)\left(\phi\left(\beta^{-1}(a) \# \beta_{\sigma} h_{1}\right) \otimes \alpha\left(h_{2}\right)\right)
\end{aligned}
$$

Thus we have

$$
\begin{aligned}
\beta^{-1}(a) \lambda\left(h_{1}\right) \#_{\sigma^{\prime}} \alpha\left(h_{2}\right) & =\sum_{i} \beta^{-1}(a) a_{i} \#_{\sigma^{\prime}} \alpha\left(h_{i}\right)=\beta^{-1}(a) \cdot\left(\sum_{i} a_{i} \# \sigma_{\sigma^{\prime}} h_{i}\right) \\
& =\beta^{-1}(a) \cdot \phi\left(1_{A} \#_{\sigma} \alpha^{-1}(h)\right)=\phi\left(\beta^{-1}(a) \cdot\left(1_{A} \#_{\sigma} \alpha^{-1}(h)\right)\right) \\
& =\phi(a \# \sigma h) .
\end{aligned}
$$

Therefore (1) is satisfied. 
Similarly, since $\phi^{-1}:\left(A \#_{\sigma^{\prime}} H, \beta \#_{\sigma^{\prime}} \alpha\right) \rightarrow\left(A \#_{\sigma} H, \beta \#_{\sigma} \alpha\right)$ is an isomorphism satisfying the same hypotheses as $\phi$, we can set

$$
\chi(h)=\left((i d \otimes \varepsilon) \circ \phi^{-1}\right)\left(1_{A} \#_{\sigma} h\right)
$$

and could conclude as above that $\phi^{-1}\left(a \#_{\sigma^{\prime}} h\right)=\beta^{-1}(a) \chi\left(h_{1}\right) \#_{\sigma} \alpha\left(h_{2}\right)$ for all $a \in A, h \in H$. Then

$$
\begin{aligned}
1_{A} \#_{\sigma} h & =\phi^{-1}\left(\phi\left(1_{A} \#_{\sigma} h\right)\right) \\
& =\phi^{-1}\left(\lambda\left(\alpha\left(h_{1}\right)\right) \#_{\sigma^{\prime}} \alpha\left(h_{2}\right)\right) \\
& =\lambda\left(h_{1}\right) \chi\left(\alpha\left(h_{21}\right)\right) \#_{\sigma} \alpha^{2}\left(h_{22}\right) .
\end{aligned}
$$

Applying $i d \otimes \varepsilon$ to both sides of the above equation, we see that $\lambda\left(h_{1}\right) \chi\left(h_{2}\right)=$ $\varepsilon(h) 1_{A}$, and thus $\lambda^{-1}=\chi$. to

Now the equation $\phi^{-1}\left(\left(a \#_{\sigma^{\prime}} h\right)\left(b \#_{\sigma^{\prime}} g\right)\right)=\phi^{-1}\left(a \#_{\sigma^{\prime}} h\right) \phi^{-1}\left(b \#_{\sigma^{\prime}} g\right)$ yields

$$
\begin{aligned}
& \left\{\beta^{-1}(a)\left[\left(\alpha^{-2}\left(h_{1}\right) \cdot^{\prime} \beta^{-3}(b)\right) \sigma^{\prime}\left(\alpha^{-1}\left(h_{21}\right), \alpha^{-2}\left(g_{1}\right)\right)\right]\right\} \\
& \quad \chi\left(\alpha^{2}\left(h_{221}\right) \alpha\left(g_{21}\right)\right) \#_{\sigma} \alpha^{3}\left(h_{222}\right) \alpha^{2}\left(g_{22}\right) \\
& =\left(\beta^{-1}(a) \chi\left(h_{1}\right)\right)\left\{\left[h_{21} \cdot^{\prime}\left(\beta^{-3}(b) \chi\left(\alpha^{-2}\left(g_{1}\right)\right)\right)\right] \sigma\left(\alpha\left(h_{221}\right), g_{21}\right)\right\} \\
& \quad \#_{\sigma} \alpha^{3}\left(h_{222}\right) \alpha^{2}\left(g_{22}\right) .
\end{aligned}
$$

Set $a=b=1_{A}$ and apply $i d \otimes \varepsilon$ to both sides of (4.2). We obtain

$$
\begin{aligned}
\sigma^{\prime}\left(h_{1}, g_{1}\right) \chi\left(h_{2} g_{2}\right) & =\chi\left(\alpha\left(h_{1}\right)\right)\left[\left(h_{21} \cdot \chi\left(\alpha^{-1}\left(g_{1}\right)\right)\right) \sigma\left(h_{22}, \alpha^{-1}\left(g_{2}\right)\right)\right] \\
& =\left[\chi\left(h_{1}\right)\left(h_{21} \cdot \chi\left(\alpha^{-1}\left(g_{1}\right)\right)\right)\right] \sigma\left(\alpha\left(h_{22}\right), g_{2}\right) \\
& =\left[\chi\left(\alpha\left(h_{11}\right)\right)\left(h_{12} \cdot \chi\left(\alpha^{-1}\left(g_{1}\right)\right)\right)\right] \sigma\left(h_{2}, g_{2}\right) .
\end{aligned}
$$

Then we have

$$
\begin{aligned}
& \left(\sigma^{\prime}\left(h_{11}, g_{11}\right) \chi\left(h_{12} g_{12}\right)\right) \lambda\left(h_{2} g_{2}\right) \\
& =\left\{\left[\chi\left(\alpha\left(h_{111}\right)\right)\left(h_{112} \cdot \chi\left(\alpha^{-1}\left(g_{11}\right)\right)\right)\right] \sigma\left(h_{12}, g_{12}\right)\right\} \lambda\left(h_{2} g_{2}\right) \\
& \Rightarrow \quad \sigma^{\prime}\left(\alpha\left(h_{11}\right), \alpha\left(g_{11}\right)\right)\left(\chi\left(h_{12} g_{12}\right) \lambda\left(\alpha^{-1}\left(h_{2} g_{2}\right)\right)\right) \\
& =\left[\chi\left(\alpha^{2}\left(h_{111}\right)\right)\left(\alpha\left(h_{112}\right) \cdot \chi\left(g_{11}\right)\right)\right]\left[\sigma\left(h_{12}, g_{12}\right) \lambda\left(\alpha^{-1}\left(h_{2} g_{2}\right)\right)\right] \\
& \Rightarrow \quad \sigma^{\prime}\left(h_{1}, g_{1}\right)\left(\chi\left(h_{21} g_{21}\right) \lambda\left(h_{22} g_{22}\right)\right) \\
& =\left[\chi\left(\alpha\left(h_{11}\right)\right)\left(h_{12} \cdot \chi\left(\alpha^{-1}\left(g_{1}\right)\right)\right)\right]\left[\sigma\left(h_{21}, g_{21}\right) \lambda\left(h_{22} g_{22}\right)\right] \\
& \Rightarrow \quad \sigma^{\prime}(h, g)=\left[\chi\left(\alpha\left(h_{11}\right)\right)\left(h_{12} \cdot \chi\left(\alpha^{-1}\left(g_{1}\right)\right)\right)\right]\left[\sigma\left(h_{21}, g_{21}\right) \lambda\left(h_{22} g_{22}\right)\right] \text {, }
\end{aligned}
$$

which implies that (3) holds.

Again use (4.2) with $a=1_{A}$ and $g=1_{H}$, and apply $i d \otimes \varepsilon$ to its both sides to see that

$$
\left(h_{1} \cdot \beta^{-1}(b)\right) \chi\left(\alpha\left(h_{2}\right)\right)=\chi\left(\alpha\left(h_{1}\right)\right)\left(h_{2} \cdot \beta^{-1}(b)\right) .
$$


EQUIVALENT CROSSED PRODUCTS OF MONOIDAL HOM-HOPF ALGEBRAS 361

Therefore,

$$
\begin{aligned}
{\left[\chi\left(\alpha\left(h_{11}\right)\right)\left(h_{12} \cdot \beta^{-1}(b)\right)\right] \lambda\left(\alpha\left(h_{2}\right)\right) } & =\left[\left(h_{11} \cdot^{\prime} \beta^{-1}(b)\right) \chi\left(\alpha\left(h_{12}\right)\right)\right] \lambda\left(\alpha\left(h_{2}\right)\right) \\
& =\left(\alpha\left(h_{11}\right) \cdot^{\prime} b\right)\left(\chi\left(\alpha\left(h_{12}\right)\right) \lambda\left(h_{2}\right)\right) \\
& =\left(h_{1} \cdot^{\prime} b\right)\left(\chi\left(\alpha\left(h_{21}\right)\right) \lambda\left(\alpha\left(h_{22}\right)\right)\right) \\
& =\left(h_{1} \cdot^{\prime} b\right) 1_{A} \varepsilon\left(h_{2}\right) \\
& =h \cdot^{\prime} \beta(b),
\end{aligned}
$$

gives (2).

The converse follows as in the proof of Proposition 4.1. Indeed, the fact that $\phi$ in (1) is an isomorphism of left $(A, \beta)$-Hom-modules, right $(H, \alpha)$ comodules is clear now, whose inverse $\psi: A \#_{\sigma^{\prime}} H \rightarrow A \#_{\sigma} H$ is defined by $\psi\left(a \# \sigma_{\sigma^{\prime}} h\right)=\beta^{-1}(a) \lambda^{-1}\left(h_{1}\right) \#_{\sigma} \alpha\left(h_{2}\right)$. And the following routine computation shows that $\psi$ is a morphism of Hom-algebras, then so is $\phi$.

$$
\begin{aligned}
& \psi\left(\left(a \#{ }_{\sigma^{\prime}} h\right)\left(b \#_{\sigma^{\prime}} g\right)\right) \\
& =\psi\left[a\left(\left(\alpha^{-1}\left(h_{1}\right) \cdot \beta^{-2}(b)\right) \sigma^{\prime}\left(h_{21}, \alpha^{-1}\left(g_{1}\right)\right)\right) \#_{\sigma^{\prime}} \alpha^{2}\left(h_{22}\right) \alpha\left(g_{2}\right)\right] \\
& =\left\{\beta^{-1}(a)\left[\left(\alpha^{-2}\left(h_{1}\right) \cdot^{\prime} \beta^{-3}(b)\right) \sigma^{\prime}\left(\alpha^{-1}\left(h_{21}\right), \alpha^{-2}\left(g_{1}\right)\right)\right]\right\} \\
& \times \lambda^{-1}\left(\alpha^{2}\left(h_{221}\right) \alpha\left(g_{21}\right)\right) \#_{\sigma} \alpha^{3}\left(h_{222}\right) \alpha^{2}\left(g_{22}\right) \\
& =\left\{\left[\beta^{-2}(a)\left(\alpha^{-2}\left(h_{1}\right) \cdot^{\prime} \beta^{-3}(b)\right)\right] \sigma^{\prime}\left(h_{21}, \alpha^{-1}\left(g_{1}\right)\right)\right\} \\
& \lambda^{-1}\left(\alpha^{2}\left(h_{221}\right) \alpha\left(g_{21}\right)\right) \#_{\sigma} \alpha^{3}\left(h_{222}\right) \alpha^{2}\left(g_{22}\right) \\
& =\left[\beta^{-1}(a)\left(\alpha^{-1}\left(h_{1}\right) \cdot^{\prime} \beta^{-2}(b)\right)\right]\left(\sigma^{\prime}\left(h_{21}, \alpha^{-1}\left(g_{1}\right)\right) \lambda^{-1}\left(\alpha\left(h_{221}\right) g_{21}\right)\right) \\
& \#_{\sigma} \alpha^{3}\left(h_{222}\right) \alpha^{2}\left(g_{22}\right) \\
& =\left[\beta^{-1}(a)\left(\alpha^{-1}\left(h_{1}\right) \cdot^{\prime} \beta^{-2}(b)\right)\right]\left\{\left[\left(\lambda^{-1}\left(\alpha\left(h_{2111}\right)\right)\left(h_{2112} \cdot \lambda^{-1}\left(\alpha^{-2}\left(g_{11}\right)\right)\right)\right)\right.\right. \\
& \left.\left.\times\left(\sigma\left(h_{2121}, \alpha^{-1}\left(g_{121}\right)\right) \lambda\left(h_{2122} \alpha^{-1}\left(g_{122}\right)\right)\right)\right] \lambda^{-1}\left(\alpha\left(h_{221}\right) g_{21}\right)\right\} \\
& \#_{\sigma} \alpha^{3}\left(h_{222}\right) \alpha^{2}\left(g_{22}\right) \\
& =\left[\beta^{-1}(a)\left(\alpha^{-1}\left(h_{1}\right) \bullet^{\prime} \beta^{-2}(b)\right)\right]\left\{\left[\lambda^{-1}\left(\alpha^{2}\left(h_{2111}\right)\right)\left(\alpha\left(h_{2112}\right) \cdot \lambda^{-1}\left(\alpha^{-1}\left(g_{11}\right)\right)\right)\right]\right. \\
& \left.\times\left[\left(\sigma\left(h_{2121}, \alpha^{-1}\left(g_{121}\right)\right) \lambda\left(h_{2122} \alpha^{-1}\left(g_{122}\right)\right)\right) \lambda^{-1}\left(h_{221} \alpha^{-1}\left(g_{21}\right)\right)\right]\right\} \\
& \#_{\sigma} \alpha^{3}\left(h_{222}\right) \alpha^{2}\left(g_{22}\right) \\
& =\left[\beta^{-1}(a)\left(\alpha^{-1}\left(h_{1}\right) \cdot^{\prime} \beta^{-2}(b)\right)\right]\left\{\left[\lambda^{-1}\left(\alpha^{2}\left(h_{2 \underline{11}}\right)\right)\left(\alpha\left(h_{2 \underline{112}}\right) \cdot \lambda^{-1}\left(\alpha^{-1}\left(g_{\underline{\underline{11}}}\right)\right)\right)\right]\right. \\
& \left.\times\left[\sigma\left(\alpha\left(h_{2 \underline{121}}\right), g_{\underline{\underline{121}}}\right)\left(\lambda\left(h_{2 \underline{122}} \alpha^{-1}\left(g_{\underline{122}}\right)\right) \lambda^{-1}\left(\alpha^{-1}\left(h_{2 \underline{21}}\right) \alpha^{-2}\left(g_{\underline{\underline{2}} 1}\right)\right)\right)\right]\right\} \\
& \#_{\sigma} \alpha^{3}\left(h_{2 \underline{22}}\right) \alpha^{2}\left(g_{\underline{2} 2}\right) \\
& =\left[\beta^{-1}(a)\left(\alpha^{-1}\left(h_{1}\right) \cdot^{\prime} \beta^{-2}(b)\right)\right]\left\{\left[\lambda^{-1}\left(\alpha\left(h_{211}\right)\right)\left(h_{212} \cdot \lambda^{-1}\left(\alpha^{-2}\left(g_{1}\right)\right)\right)\right]\right.
\end{aligned}
$$




$$
\begin{aligned}
& \left.\times\left[\sigma\left(\alpha\left(h_{22 \underline{11}}\right), g_{2 \underline{\underline{11}}}\right)\left(\lambda\left(h_{22 \underline{12}} \alpha^{-1}\left(g_{2 \underline{\underline{12}}}\right)\right) \lambda^{-1}\left(h_{22 \underline{21}} \alpha^{-1}\left(g_{2 \underline{\underline{2}} 1}\right)\right)\right)\right]\right\} \\
& \#_{\sigma} \alpha^{4}\left(h_{22 \underline{2} 2}\right) \alpha^{3}\left(g_{2 \underline{2} 2}\right) \\
& =\left[\beta^{-1}(a)\left(\alpha^{-1}\left(h_{1}\right) \cdot^{\prime} \beta^{-2}(b)\right)\right]\left\{\left[\lambda^{-1}\left(\alpha\left(h_{211}\right)\right)\left(h_{212} \cdot \lambda^{-1}\left(\alpha^{-2}\left(g_{1}\right)\right)\right)\right]\right. \\
& \left.\times\left[\sigma\left(h_{221}, \alpha^{-1}\left(g_{21}\right)\right)\left(\lambda\left(h_{222 \underline{1}} \alpha^{-1}\left(g_{22 \underline{1}}\right)\right) \lambda^{-1}\left(\alpha\left(h_{222 \underline{12}}\right) g_{22 \underline{21}}\right)\right)\right]\right\} \\
& \#_{\sigma} \alpha^{5}\left(h_{222 \underline{22}}\right) \alpha^{4}\left(g_{22 \underline{22}}\right) \\
& =\left[\beta^{-1}(a)\left(\alpha^{-1}\left(h_{1}\right) \cdot^{\prime} \beta^{-2}(b)\right)\right]\left\{\left[\lambda^{-1}\left(\alpha\left(h_{211}\right)\right)\left(h_{212} \cdot \lambda^{-1}\left(\alpha^{-2}\left(g_{1}\right)\right)\right)\right]\right. \\
& \left.\times\left[\sigma\left(h_{221}, \alpha^{-1}\left(g_{21}\right)\right)\left(\underline{\lambda\left(\alpha\left(h_{22211}\right) g_{2211}\right) \lambda^{-1}\left(\alpha\left(h_{22212}\right) g_{2212}\right)}\right)\right]\right\} \\
& \#_{\sigma} \alpha^{4}\left(h_{2222}\right) \alpha^{3}\left(g_{222}\right) \\
& =\left[\beta^{-1}(a)\left(\alpha^{-1}\left(h_{1}\right) \cdot^{\prime} \beta^{-2}(b)\right)\right]\left\{\left[\lambda^{-1}\left(\alpha\left(h_{211}\right)\right)\left(h_{212} \cdot \lambda^{-1}\left(\alpha^{-2}\left(g_{1}\right)\right)\right)\right]\right. \\
& \left.\times \sigma\left(\alpha\left(h_{221}\right), g_{21}\right)\right\} \#_{\sigma} \alpha^{3}\left(h_{222}\right) \alpha^{2}\left(g_{22}\right) \\
& =\left\{\beta^{-1}(a)\left[\left(\lambda^{-1}\left(h_{111}\right)\left(\alpha^{-1}\left(h_{112}\right) \cdot \beta^{-4}(b)\right)\right) \lambda\left(h_{12}\right)\right]\right\} \\
& \times\left\{\lambda^{-1}\left(\alpha^{2}\left(h_{211}\right)\right)\left[\left(h_{212} \cdot \lambda^{-1}\left(\alpha^{-2}\left(g_{1}\right)\right)\right) \sigma\left(h_{221}, \alpha^{-1}\left(g_{21}\right)\right)\right]\right\} \\
& \#_{\sigma} \alpha^{3}\left(h_{222}\right) \alpha^{2}\left(g_{22}\right) \\
& =\left\{\left[\beta^{-2}(a)\left(\lambda^{-1}\left(h_{111}\right)\left(\alpha^{-1}\left(h_{112}\right) \cdot \beta^{-4}(b)\right)\right)\right] \lambda\left(\alpha\left(h_{12}\right)\right)\right\} \\
& \times\left\{\lambda^{-1}\left(\alpha^{2}\left(h_{211}\right)\right)\left[\left(h_{212} \cdot \lambda^{-1}\left(\alpha^{-2}\left(g_{1}\right)\right)\right) \sigma\left(h_{221}, \alpha^{-1}\left(g_{21}\right)\right)\right]\right\} \\
& \#_{\sigma} \alpha^{3}\left(h_{222}\right) \alpha^{2}\left(g_{22}\right) \\
& =\left\{\beta^{-1}(a)\left[\lambda^{-1}\left(\alpha\left(h_{111}\right)\right)\left(h_{112} \cdot \beta^{-3}(b)\right)\right]\right\}\left\{\lambda ( \alpha ( h _ { 1 2 } ) ) \left[\lambda^{-1}\left(\alpha\left(h_{211}\right)\right)\right.\right. \\
& \left.\left.\times\left(\left(\alpha^{-1}\left(h_{212}\right) \cdot \lambda^{-1}\left(\alpha^{-3}\left(g_{1}\right)\right)\right) \sigma\left(\alpha^{-1}\left(h_{221}\right), \alpha^{-2}\left(g_{21}\right)\right)\right)\right]\right\} \\
& \#_{\sigma} \alpha^{3}\left(h_{222}\right) \alpha^{2}\left(g_{22}\right) \\
& =\left\{\beta^{-1}(a)\left[\lambda^{-1}\left(\alpha\left(h_{\underline{111}}\right)\right)\left(h_{\underline{112}} \cdot \beta^{-3}(b)\right)\right]\right\}\left\{\left(\lambda\left(h_{\underline{12}}\right) \lambda^{-1}\left(\alpha\left(h_{\underline{211}}\right)\right)\right)\right. \\
& \left.\times\left[\left(h_{212} \cdot \lambda^{-1}\left(\alpha^{-2}\left(g_{1}\right)\right)\right) \sigma\left(h_{221}, \alpha^{-1}\left(g_{21}\right)\right)\right]\right\} \#_{\sigma} \alpha^{3}\left(h_{222}\right) \alpha^{2}\left(g_{22}\right) \\
& =\left\{\beta^{-1}(a)\left[\lambda^{-1}\left(h_{11}\right)\left(\alpha^{-1}\left(h_{12}\right) \cdot \beta^{-3}(b)\right)\right]\right\}\left\{\left(\lambda\left(h_{21}\right) \lambda^{-1}\left(\alpha^{2}\left(h_{2 \underline{211}}\right)\right)\right)\right. \\
& \left.\times\left[\left(\alpha\left(h_{2 \underline{212}}\right) \cdot \lambda^{-1}\left(\alpha^{-2}\left(g_{1}\right)\right)\right) \sigma\left(\alpha\left(h_{2 \underline{22} 1}\right), \alpha^{-1}\left(g_{21}\right)\right)\right]\right\} \\
& \#_{\sigma} \alpha^{4}\left(h_{2 \underline{22} 2}\right) \alpha^{2}\left(g_{22}\right) \\
& =\left\{\beta^{-1}(a)\left[\lambda^{-1}\left(h_{11}\right)\left(\alpha^{-1}\left(h_{12}\right) \cdot \beta^{-3}(b)\right)\right]\right\}\left\{\left(\lambda\left(\alpha\left(h_{21 \underline{1}}\right)\right) \lambda^{-1}\left(\alpha^{2}\left(h_{21 \underline{21}}\right)\right)\right)\right.
\end{aligned}
$$


EQUIVALENT CROSSED PRODUCTS OF MONOIDAL HOM-HOPF ALGEBRAS 363

$$
\begin{aligned}
& \left.\times\left[\left(\alpha\left(h_{21 \underline{22}}\right) \cdot \lambda^{-1}\left(\alpha^{-2}\left(g_{1}\right)\right)\right) \sigma\left(h_{221}, \alpha^{-1}\left(g_{21}\right)\right)\right]\right\} \#_{\sigma} \alpha^{3}\left(h_{222}\right) \alpha^{2}\left(g_{22}\right) \\
& =\left\{\beta^{-1}(a)\left[\lambda^{-1}\left(h_{11}\right)\left(\alpha^{-1}\left(h_{12}\right) \cdot \beta^{-3}(b)\right)\right]\right\}\left\{\left(\underline{\lambda\left(\alpha^{2}\left(h_{2111}\right)\right) \lambda^{-1}\left(\alpha^{2}\left(h_{2112}\right)\right)}\right)\right. \\
& \left.\times\left[\left(h_{212} \cdot \lambda^{-1}\left(\alpha^{-2}\left(g_{1}\right)\right)\right) \sigma\left(h_{221}, \alpha^{-1}\left(g_{21}\right)\right)\right]\right\} \#_{\sigma} \alpha^{3}\left(h_{222}\right) \alpha^{2}\left(g_{22}\right) \\
& =\left[\left(\beta^{-2}(a) \lambda^{-1}\left(h_{11}\right)\right)\left(h_{12} \cdot \beta^{-2}(b)\right)\right]\left[\left(h_{21} \cdot \lambda^{-1}\left(\alpha^{-1}\left(g_{1}\right)\right)\right)\right. \\
& \left.\times \sigma\left(\alpha\left(h_{221}\right), g_{21}\right)\right] \#_{\sigma} \alpha^{3}\left(h_{222}\right) \alpha^{2}\left(g_{22}\right) \\
& =\left(\beta^{-1}(a) \lambda^{-1}\left(\alpha\left(h_{11}\right)\right)\right)\left\{( h _ { 1 2 } \cdot \beta ^ { - 2 } ( b ) ) \left[\left(\alpha^{-1}\left(h_{21}\right) \cdot \lambda^{-1}\left(\alpha^{-2}\left(g_{1}\right)\right)\right)\right.\right. \\
& \left.\left.\times \sigma\left(h_{221}, \alpha^{-1}\left(g_{21}\right)\right)\right]\right\} \#_{\sigma} \alpha^{3}\left(h_{222}\right) \alpha^{2}\left(g_{22}\right) \\
& =\left(\beta^{-1}(a) \lambda^{-1}\left(\alpha\left(h_{\underline{11}}\right)\right)\right)\left\{\left[\left(\alpha^{-1}\left(h_{\underline{12}}\right) \cdot \beta^{-3}(b)\right)\left(\alpha^{-1}\left(h_{\underline{2} 1}\right) \cdot \lambda^{-1}\left(\alpha^{-2}\left(g_{1}\right)\right)\right)\right]\right. \\
& \left.\times \sigma\left(\alpha\left(h_{\underline{2} 21}\right), g_{21}\right)\right\} \#_{\sigma} \alpha^{3}\left(h_{222}\right) \alpha^{2}\left(g_{22}\right) \\
& =\left(\beta^{-1}(a) \lambda^{-1}\left(h_{1}\right)\right)\left\{\left[\left(\alpha^{-1}\left(h_{2 \underline{1}}\right) \cdot \beta^{-3}(b)\right)\left(h_{2 \underline{21}} \cdot \lambda^{-1}\left(\alpha^{-2}\left(g_{1}\right)\right)\right)\right]\right. \\
& \left.\times \sigma\left(\alpha^{2}\left(h_{2 \underline{221}}\right), g_{21}\right)\right\} \#_{\sigma} \alpha^{4}\left(h_{2 \underline{22} 2}\right) \alpha^{2}\left(g_{22}\right) \\
& =\left(\beta^{-1}(a) \lambda^{-1}\left(h_{1}\right)\right)\left\{\left[\left(h_{211} \cdot \beta^{-3}(b)\right)\left(h_{212} \cdot \lambda^{-1}\left(\alpha^{-2}\left(g_{1}\right)\right)\right)\right]\right. \\
& \left.\times \sigma\left(\alpha\left(h_{221}\right), g_{21}\right)\right\} \#{ }_{\sigma} \alpha^{3}\left(h_{222}\right) \alpha^{2}\left(g_{22}\right) \\
& \stackrel{(3.3)}{=}\left(\beta^{-1}(a) \lambda^{-1}\left(h_{1}\right)\right)\left\{\left[h_{21} \cdot\left(\beta^{-3}(b) \lambda^{-1}\left(\alpha^{-2}\left(g_{1}\right)\right)\right)\right] \sigma\left(\alpha\left(h_{221}\right), g_{21}\right)\right\} \\
& \#_{\sigma} \alpha^{3}\left(h_{222}\right) \alpha^{2}\left(g_{22}\right) \\
& =\left(\beta^{-1}(a) \lambda^{-1}\left(h_{1}\right) \#_{\sigma} \alpha\left(h_{2}\right)\right)\left(\beta^{-1}(b) \lambda^{-1}\left(g_{1}\right) \#_{\sigma} \alpha\left(g_{2}\right)\right) \\
& =\psi\left(a \# \sigma^{\prime} h\right) \psi\left(b \# \sigma_{\sigma^{\prime}} g\right) \text {. }
\end{aligned}
$$

Hence, we complete the proof of this theorem.

REMARK 4.4. With a same assumption as in Theorem 4.3, if we let the weak Hom-action on $(A, \beta)$ be trivial, then the weak action on $\left(A^{\prime}, \beta^{\prime}\right)$ becomes inner, and the cocycle $\sigma^{\prime}$ is just the map $\tau$ defined in Proposition 4.1.

Theorem 4.3 suggests the following definition:

Definition 4.5. Let $(A, \beta)$ be a monoidal Hom-algebra and $(H, \alpha)$ a monoidal Hom-Hopf algebra. Two Hom-crossed products $\left(A \#_{\sigma} H, \beta \#_{\sigma} \alpha\right)$ and $\left(A \# \sigma_{\sigma^{\prime}} H, \beta \# \sigma_{\sigma^{\prime}} \alpha\right)$ (or two Hom-crossed systems $(\cdot, \sigma)$ and $\left(\cdot^{\prime}, \sigma^{\prime}\right)$ for $(H, \alpha)$ over $(A, \beta))$ are equivalent if there exists a Hom-algebra isomorphism $\phi:\left(A \#_{\sigma} H, \beta \#_{\sigma} \alpha\right) \rightarrow\left(A \#_{\sigma^{\prime}} H, \beta \#_{\sigma^{\prime}} \alpha\right)$ which is a left $(A, \beta)$-Hom-module, right $(H, \alpha)$-Hom-comodule morphism. 
We denote the equivalent Hom-crossed products by $\left(A \#_{\sigma} H, \beta \#_{\sigma} \alpha\right) \sim$ $\left(A \# \sigma_{\sigma^{\prime}} H, \beta \#{ }_{\sigma^{\prime}} \alpha\right)$, and the equivalent Hom-crossed systems for $(H, \alpha)$ over $(A, \beta)$ by $(\cdot, \sigma) \sim\left({ }^{\prime}, \sigma^{\prime}\right)$, respectively.

Definition 4.6 ([20]). A left lazy Hom-2-cocycle on a monoidal Hombialgebra $(H, \alpha)$ is a linear map $\digamma:(H \otimes H, \alpha \otimes \alpha) \rightarrow k$ in $\widetilde{\mathcal{H}}\left(\mathcal{M}_{k}\right)$ (i.e., $\digamma \circ(\alpha \otimes \alpha)=\digamma)$ satisfying

$$
\begin{gathered}
\digamma\left(1_{H}, x\right)=\varepsilon(x)=\digamma\left(x, 1_{H}\right), \\
\digamma\left(x_{1}, y_{1}\right) \digamma\left(x_{2} y_{2}, z\right)=\digamma\left(y_{1}, z_{1}\right) \digamma\left(x, y_{2} z_{2}\right), \\
\digamma\left(x_{1}, y_{1}\right) x_{2} y_{2}=x_{1} y_{1} \digamma\left(x_{2}, y_{2}\right),
\end{gathered}
$$

for all $x, y, z \in H$.

Lemma 4.7 ([20]). The set of convolution invertible lazy Hom-2-cocycles denoted by $Z_{L}^{2}(H, \alpha)$ is a group under convolution product.

Lemma 4.8 ([20]). Let $\gamma:(H, \alpha) \rightarrow k$ be a convolution invertible linear map in $\widetilde{\mathcal{H}}\left(\mathcal{M}_{k}\right)$ such that $\gamma\left(1_{H}\right)=1_{k}$, and define

$$
D^{1}(\gamma):(H \otimes H, \alpha \otimes \alpha) \rightarrow k, \quad D^{1}(\gamma)(h, g)=\gamma\left(h_{1}\right) \gamma\left(g_{1}\right) \gamma^{-1}\left(h_{2} g_{2}\right),
$$

for all $h, g \in H$. Then $D^{1}(\gamma) \in Z_{L}^{2}(H, \alpha)$.

Proof. It is a straightforward consequence of Theorem 3.4 by letting $(A, \beta)=(H, \alpha)$ and the weak action is trivial.

The set of all convolution invertible linear maps $\gamma:(H, \alpha) \rightarrow k$ in $\widetilde{\mathcal{H}}\left(\mathcal{M}_{k}\right)$ satisfying

$$
\gamma\left(h_{1}\right) h_{2}=h_{1} \gamma\left(h_{2}\right), \quad \gamma\left(1_{H}\right)=1_{k}, \quad \forall h \in H,
$$

is denoted by $\operatorname{Reg}_{L}^{1}(H, \alpha)$, which is a group under convolution product.

Proposition 4.9 ([20]). The map $D^{1}: \operatorname{Reg}_{L}^{1}(H, \alpha) \rightarrow Z_{L}^{2}(H, \alpha)$ is a group homomorphism, whose image denoted by $B_{L}^{2}(H, \alpha)$, is contained in the center of $Z_{L}^{2}(H, \alpha)$. We call the quotient group

$$
H_{L}^{2}(H, \alpha):=Z_{L}^{2}(H, \alpha) / B_{L}^{2}(H, \alpha)
$$

the second Hom-lazy cohomology group of $(H, \alpha)$.

Proposition 4.10. Assume that $(H, \alpha)$ is a monoidal Hom-Hopf algebra, and $\left(B,\left.\beta\right|_{B}\right) \subseteq(A, \beta)$ is an $(H, \alpha)$-cleft extension, and let $(\cdot, \sigma)$ be the corresponding Hom-crossed system for $(H, \alpha)$ over $\left(B,\left.\beta\right|_{B}\right)$. Then we have the following.

(1) Let $\digamma \in Z_{L}^{2}(H, \alpha)$. Then $(\cdot,(\beta \circ \sigma) * \digamma)$ is also a Hom-crossed system for $(H, \alpha)$ over $(A, \beta)$.

(2) Let $\digamma, \digamma^{\prime} \in Z_{L}^{2}(H, \alpha)$. If $\digamma^{-1} * \digamma^{\prime}=D^{1}(\gamma)$ for some $\gamma \in \operatorname{Reg}_{L}^{1}(H, \alpha)$, then $(\cdot,(\beta \circ \sigma) * \digamma) \sim\left(\cdot,(\beta \circ \sigma) * \digamma^{\prime}\right)$. 
EQUIVALENT CROSSED PRODUCTS OF MONOIDAL HOM-HOPF ALGEBRAS 365

Proof. (1) First, we show that (3.5) for the map $(\beta \circ \sigma) * \digamma$ holds. For all $h, g \in H$ and $b \in B$,

$$
\begin{aligned}
((\beta \circ \sigma) & * \digamma)\left(\alpha\left(h_{1}\right), \alpha\left(g_{1}\right)\right)\left(\left(h_{2} g_{2}\right) \cdot b\right) \\
\quad= & \sigma\left(\alpha^{2}\left(h_{11}\right), \alpha^{2}\left(g_{11}\right)\right) \digamma\left(\alpha\left(h_{12}\right), \alpha\left(g_{12}\right)\right)\left(\left(h_{2} g_{2}\right) \cdot b\right) \\
& =\sigma\left(\alpha\left(h_{1}\right), \alpha\left(g_{1}\right)\right) \digamma\left(h_{21}, g_{21}\right)\left(\alpha\left(h_{22} g_{22}\right) \cdot b\right) \\
& \stackrel{(4.5)}{=} \sigma\left(\alpha\left(h_{1}\right), \alpha\left(g_{1}\right)\right) \digamma\left(h_{22}, g_{22}\right)\left(\alpha\left(h_{21} g_{21}\right) \cdot b\right) \\
& =\sigma\left(\alpha^{2}\left(h_{11}\right), \alpha^{2}\left(g_{11}\right)\right)\left(\alpha\left(h_{12} g_{12}\right) \cdot b\right) \digamma\left(h_{2}, g_{2}\right) \\
& \quad \stackrel{(3.5)}{=}\left(\alpha^{2}\left(h_{11}\right) \cdot\left(\alpha\left(g_{11}\right) \cdot \beta^{-1}(b)\right)\right) \sigma\left(\alpha^{2}\left(h_{12}\right), \alpha^{2}\left(g_{12}\right)\right) \digamma\left(h_{2}, g_{2}\right) \\
& =\left(\alpha\left(h_{1}\right) \cdot\left(g_{1} \cdot \beta^{-1}(b)\right)\right) \sigma\left(\alpha^{2}\left(h_{21}\right), \alpha^{2}\left(g_{21}\right)\right) \digamma\left(\alpha\left(h_{22}\right), \alpha\left(g_{22}\right)\right) \\
& =\left(\alpha\left(h_{1}\right) \cdot\left(g_{1} \cdot \beta^{-1}(b)\right)\right)((\beta \circ \sigma) * \digamma)\left(\alpha\left(h_{2}\right), \alpha\left(g_{2}\right)\right) .
\end{aligned}
$$

Second, we show that (3.6) for the map $(\beta \circ \sigma) * \digamma$ holds. For all $x, y, z \in H$,

$$
\begin{aligned}
&((\beta \circ \sigma) * \digamma)\left(\alpha\left(x_{1}\right), \alpha\left(y_{1}\right)\right)((\beta \circ \sigma) * \digamma)\left(x_{2} y_{2}, z\right) \\
&=\sigma\left(\alpha^{2}\left(x_{11}\right), \alpha^{2}\left(y_{11}\right)\right) \digamma\left(x_{12}, y_{12}\right) \sigma\left(\alpha\left(x_{21} y_{21}\right), \alpha\left(z_{1}\right)\right) \digamma\left(x_{22} y_{22}, z_{2}\right) \\
&= \sigma\left(\alpha\left(x_{1}\right), \alpha\left(y_{1}\right)\right) \digamma\left(x_{21}, y_{21}\right) \sigma\left(\alpha^{2}\left(x_{221} y_{221}\right), \alpha\left(z_{1}\right)\right) \digamma\left(\alpha\left(x_{222} y_{222}\right), z_{2}\right) \\
&= \sigma\left(\alpha\left(x_{1}\right), \alpha\left(y_{1}\right)\right) \digamma\left(x_{211}, y_{211}\right) \sigma\left(\alpha^{2}\left(x_{212} y_{212}\right), \alpha\left(z_{1}\right)\right) \digamma\left(x_{22} y_{22}, z_{2}\right) \\
& \stackrel{(4.5)}{=} \sigma\left(\alpha\left(x_{1}\right), \alpha\left(y_{1}\right)\right) \digamma\left(x_{212}, y_{212}\right) \sigma\left(\alpha^{2}\left(x_{211} y_{211}\right), \alpha\left(z_{1}\right)\right) \digamma\left(x_{22} y_{22}, z_{2}\right) \\
&=\sigma\left(\alpha\left(x_{1}\right), \alpha\left(y_{1}\right)\right) \digamma\left(x_{221}, y_{221}\right) \sigma\left(\alpha\left(x_{21} y_{21}\right), \alpha\left(z_{1}\right)\right) \digamma\left(\alpha\left(x_{222} y_{222}\right), z_{2}\right) \\
&=\sigma\left(\alpha^{2}\left(x_{11}\right), \alpha^{2}\left(y_{11}\right)\right) \digamma\left(x_{21}, y_{21}\right) \sigma\left(\alpha\left(x_{12} y_{12}\right), \alpha\left(z_{1}\right)\right) \digamma\left(x_{22} y_{22}, z_{2}\right) \\
& \stackrel{(3.6)}{=}\left(\alpha^{2}\left(x_{11}\right) \cdot \sigma\left(\alpha\left(y_{11}\right), \alpha\left(z_{11}\right)\right)\right) \digamma\left(x_{21}, y_{21}\right) \sigma\left(\alpha^{2}\left(x_{12}\right), \alpha\left(y_{12} z_{12}\right)\right) \\
& \digamma\left(x_{22} y_{22}, z_{2}\right) \\
&(\stackrel{4.4}{=})\left(\alpha^{2}\left(x_{11}\right) \cdot \sigma\left(\alpha\left(y_{11}\right), \alpha\left(z_{11}\right)\right)\right) \digamma\left(y_{21}, z_{21}\right) \sigma\left(\alpha^{2}\left(x_{12}\right), \alpha\left(y_{12} z_{12}\right)\right) \\
& \digamma\left(x_{2}, y_{22} z_{22}\right) \\
&=\left(\alpha\left(x_{1}\right) \cdot \sigma\left(y_{1}, z_{1}\right)\right) \digamma\left(y_{221}, z_{221}\right) \sigma\left(\alpha^{2}\left(x_{21}\right), \alpha\left(y_{21} z_{21}\right)\right) \\
& \digamma\left(x_{22}, y_{222} z_{222}\right) \\
&=\left(\alpha\left(x_{1}\right) \cdot \sigma\left(y_{1}, z_{1}\right)\right) \digamma\left(y_{212}, z_{212}\right) \sigma\left(\alpha^{2}\left(x_{21}\right), \alpha^{2}\left(y_{211} z_{211}\right)\right) \\
& \digamma \digamma\left(x_{22}, \alpha^{-1}\left(y_{22} z_{22}\right)\right) \\
&=\left(\alpha\left(x_{1}\right) \cdot \sigma\left(y_{1}, z_{1}\right)\right) \digamma\left(y_{211}, z_{211}\right) \sigma\left(\alpha^{2}\left(x_{21}\right), \alpha^{2}\left(y_{212} z_{212}\right)\right) \\
& \digamma\left(\alpha\left(x_{22}\right), y_{22} z_{22}\right) \\
&
\end{aligned}
$$




$$
\begin{aligned}
= & \left(\alpha\left(x_{1}\right) \cdot \sigma\left(\alpha\left(y_{11}\right), \alpha\left(z_{11}\right)\right)\right) \digamma\left(y_{121}, z_{121}\right) \sigma\left(\alpha^{2}\left(x_{21}\right), \alpha^{2}\left(y_{122} z_{122}\right)\right) \\
& \digamma\left(\alpha\left(x_{22}\right), \alpha^{-1}\left(y_{2} z_{2}\right)\right) \\
= & \left(\alpha\left(x_{1}\right) \cdot \sigma\left(\alpha^{2}\left(y_{111}\right), \alpha^{2}\left(z_{111}\right)\right)\right) \digamma\left(y_{112}, z_{112}\right) \sigma\left(\alpha^{2}\left(x_{21}\right), \alpha\left(y_{12} z_{12}\right)\right) \\
& \digamma\left(\alpha\left(x_{22}\right), \alpha^{-1}\left(y_{2} z_{2}\right)\right) \\
= & \left(\alpha\left(x_{1}\right) \cdot \sigma\left(\alpha\left(y_{11}\right), \alpha\left(z_{11}\right)\right)\right) \digamma\left(y_{12}, z_{12}\right) \sigma\left(\alpha^{2}\left(x_{21}\right), \alpha\left(y_{21} z_{21}\right)\right) \\
& \digamma\left(\alpha\left(x_{22}\right), y_{22} z_{22}\right) \\
= & \left.\left.\left(\alpha\left(x_{1}\right) \cdot(\beta \circ \sigma) * \digamma\right)\left(y_{1}, z_{1}\right)\right)(\beta \circ \sigma) * \digamma\right)\left(\alpha\left(x_{2}\right), y_{2} z_{2}\right) .
\end{aligned}
$$

Last, it is obvious that the map $(\beta \circ \sigma) * \digamma$ is normal.

(2) Define $\lambda:(H, \alpha) \rightarrow\left(B,\left.\beta\right|_{B}\right), \lambda(h)=\gamma^{-1}(h) 1_{A}$. Then a direct computation shows that Theorem 4.3 (2) and (3) hold by (4.5) and (4.6). Hence $i d:\left(A \#_{\sigma} H, \beta \#_{\sigma} \alpha\right) \rightarrow\left(A \#_{\sigma^{\prime}} H, \beta \#_{\sigma^{\prime}} \alpha\right)$ is an isomorphism of Hom-algebras, which is a left $(A, \beta)$-Hom-module, right $(H, \alpha)$-Hom-comodule morphism by Theorem 4.3. Therefore, $(\cdot,(\beta \circ \sigma) * \digamma) \sim\left(\cdot,(\beta \circ \sigma) * \digamma^{\prime}\right)$.

\section{ACKNOWLEDGEMENTS.}

The authors are supported by the National Natural Science Foundation of China (Grant No. 11601203, 11571173), Natural Science Foundation of Jiangsu Province (Grant No. BK20150113), the China Postdoctoral Science Foundation (Grant No. 2018M642128), and Qing Lan Project of Jiangsu Province.

\section{REFERENCES}

[1] R. Blattner and S. Montgomery, Crossed products and Galois extensions of Hopf algebra, Pacific J. Math. 137 (1989), 37-54.

[2] S. Caenepeel and I. Goyvaerts, Monoidal Hom-Hopf algebras, Comm. Algebra 39 (2011), 2216-2240.

[3] Y. Y. Chen and L. Y. Zhang, Hom-Yang-Baxter equations and Frobenius monoidal Hom-algebras, Adv. Math. Phys. 2018, 2912578.

[4] Y. Y. Chen and L. Y. Zhang, The structure and construction of bi-Frobenius Homalgebras, Comm. Algebra 45 (2017), 2142-2162.

[5] Y. Y. Chen, Z. W. Wang and L. Y. Zhang, The fundamental theorem and Maschke's theorem in the category of relative Hom-Hopf modules, Colloq. Math. 144 (2016), $55-71$.

[6] Y. Y. Chen, Z. W. Wang and L. Y. Zhang, Integrals for monoidal Hom-Hopf algebras and their applications, J. Math. Phys. 54 (2013), 073515.

[7] Y. Y. Chen and L. Y. Zhang, Hopf-Galois extensions for monoidal Hom-Hopf algebras, Colloq. Math. 143 (2016), 127-147.

[8] Y. Doi, Equivalent crossed products for a Hopf algebra, Comm. Algebra 17 (1989), 3053-3085.

[9] A. Gohr, On Hom-algebras with surjective twisting, J. Algebra 324 (2010), 14831491. 
[10] J. T. Hartwig, D. Larsson and S. D. Silvestrov, Deformations of Lie algebras using $\sigma$-derivations, J. Algebra 295 (2006), 314-361.

[11] D. Larsson and S. D. Silvestrov, Quasi-deformations of $\operatorname{sl}_{2}(F)$ using twisted derivations, Comm. Algebra 35 (2007), 4303-4318.

[12] D. W. Lu and S. H. Wang, Crossed product and Galois extension of monoidal HomHopf algebras, arXiv:1405.7528.

[13] A. Makhlouf and S. D. Silvestrov, Hom-algebras structures, J. Gen. Lie Theory Appl. 2 (2008), 51-64.

[14] A. Makhlouf and S. Silvestrov, Hom-algebras and Hom-coalgebras, J. Algebra Appl. 9 (2010), 553-589.

[15] Z. W. Wang, Y. Y. Chen and L. Y. Zhang, Separable extensions for crossed products over monoidal Hom-Hopf algebras, J. Algebra Appl. 17 (2018), 1850161.

[16] Z. W. Wang, Y. Y. Chen and L. Y. Zhang, The antipode and Drinfel'd double of a Hom-Hopf algebra, Sci. China Math. 42 (2012), 1079-1093.

[17] D. Yau, Hom-algebras and homology, J. Lie Theory 19 (2009), 409-421.

[18] D. Yau, The Hom-Yang-Baxter equation, Hom-Lie algebras, and quasi-triangular bialgebras, J. Phys. A 42 (2009), 165202.

[19] D. Yau, Hom-bialgebras and comodule Hom-algebras, Int. Electron. J. Algebra 8 (2010), 45-64.

[20] X. F. Zhao and X. H. Zhang, Lazy 2-cocycles over monoidal Hom-Hopf algebras, Colloq. Math. 142 (2016), 61-81.

Z. Wang

College of Science

Jinling Institute of Technology

211169, Nanjing, Jiangsu

P.R. China

L. Zhang

College of Science

Nanjing Agricultural University

210095, Nanjing, Jiangsu

P.R. China

E-mail: zlyun@njau.edu.cn

H. Zheng

College of Science

Nanjing Agricultural University

210095, Nanjing, Jiangsu

P.R. China

Received: 27.10 .2018 .

Revised: 14.4.2019. 